\title{
Local Normality in Quantum Statistical Mechanics
}

\author{
Masamichi Takesaki \\ Department of Mathematics, University of California, Los Angeles, USA \\ Marinus Winnink \\ Institute for Theoretical Physics, University of Groningen, Groningen, The Netherlands
}

Received October 2, 1972

\begin{abstract}
It is shown that K.M.S.-states are locally normal on a great number of $C^{*}$-algebras that may be of interest in Quantum Statistical Mechanics. The lattice structure and the Choquet-simplex structure of various sets of states are investigated. In this respect special attention is payed to the interplay of the K.M.S.-automorphism group with other automorphism groups under whose action K.M.S.-states are possibly invariant. A seemingly weaker notion than $G$-abelianness of the algebra of observables, namely $G^{\prime}$ abelianness, is introduced and investigated. Finally a necessary and sufficient condition (on a $C^{*}$-algebra with a sequential separable factor funnel) for decomposition of a locally normal state into locally normal states is given.
\end{abstract}

\section{§ 1. Introduction}

The investigation of representations of the commutation relations and anticommutation relations by Dell'Antonio, Doplicher and Ruelle [3] has forced upon us the concept of a locally normal state. Due to this origin, locally normal states have been studied on a $C^{*}$-algebra which is a $C^{*}$-inductive limit of sub- $C^{*}$-algebras, which in essence are irreducible $C^{*}$-algebras on suitably chosen Hilbert-spaces $[14,21]$.

In [12] the concept of a locally normal state has been generalized for a $C^{*}$-algebra that is a $C^{*}$-inductive limit of a net of von Neumann algebras. In the case where the net consists of factors each having a representation on a separable Hilbert space, the net is called a funnel. We shall use the word funnel for the net of von Neumann (or rather $W^{*}$ )algebras that generate a $C^{*}$-algebra $\mathfrak{U}$ in the sense of [12], Definition 2, even if the net does not contain only factors and even if the net contains factors which are not of the same type.

It is the aim of this paper to investigate the locally normal character of K.M.S. states on a $C^{*}$-algebra $\mathfrak{U}$ with a funnel whose components are $\sigma$-finite properly infinite $W^{*}$-algebras. As a result we find then that every K.M.S. state on $\mathfrak{U}$ is locally normal. It then follows that $\omega$ is normal on every finite factor contained in $\mathfrak{U}$ and on every $\sigma$-finite properly 
infinite $W^{*}$-algebra contained in $\mathfrak{U}$. If we further specialize the funnel that generates $\mathfrak{U}$, to a sequential funnel of properly infinite $W^{*}$-algebras with a separable predual ${ }^{1}$, then the cyclic representation induced by a K.M.S. state acts on a separable Hilbert-space and consequently every K.M.S. state is normal on every factor contained in $\mathfrak{U}$. Also here $\omega$ is normal on more general $W^{*}$-algebras than factors in $\mathfrak{U}$, namely on every $\sigma$-finite properly infinite $W^{*}$-algebra contained in $\mathfrak{U}$.

In the case of a sequential funnel of properly infinite $W^{*}$-algebras with a separable predual we prove that every non-primary K.M.S. state admits a unique decomposition into extremal K.M.S. states under suitable conditions on the K.M.S. automorphism, namely that the set of K.M.S. states for a given K.M.S. automorphism at a given temperature is compact in the weak*-topology on $\mathfrak{U}^{*}$. In the course of the argument we show that every weak*-compact subset of the set of locally normal states is metrizable compact.

The simplex structure of various sets of states is investigated. For example, we shall show that the set of K.M.S. states which are also invariant under the action of an arbitrary group of automorphisms that is different from the K.M.S. automorphism group is a simplex. Furthermore we shall investigate to what extent a locally normal state can be decomposed into locally normal states.

\section{§ 2. Notation, Definitions and Preliminary Results}

Let $\mathfrak{M}$ be a $W^{*}$-algebra, then by definition $\mathfrak{M}$ is the Banach-space dual of a Banach-space $\mathfrak{M}_{*}$, i.e. $\mathfrak{M}=\left(\mathfrak{M}_{*}\right)^{*} \cdot \mathfrak{M}_{*}$ is called the predual of $\mathfrak{M}$ and consists precisely of those elements in $\mathfrak{M}^{*}$ (the Banach-space dual of $\mathfrak{M})$ which are $\sigma\left(\mathfrak{M}, \mathfrak{M}_{*}\right)$ continuous.

Because $\mathfrak{M}=\left(\mathfrak{M}_{*}\right)^{*}$, the $\sigma\left(\mathfrak{M}, \mathfrak{M}_{*}\right)$ topology on $\mathfrak{M}$ is the weak*topology on $\mathfrak{M} . \mathfrak{M}$ is said to be separable, whenever the Banach-space $\mathfrak{M}_{*}$ is separable. For $\mathfrak{M}$ separable the unit ball in $\mathfrak{M}$ is metrizable and since it is compact in the $\sigma\left(\mathfrak{M}, \mathfrak{M}_{*}\right)$ topology it is separable; we can therefore find a separable $C^{*}$-algebra $\mathfrak{A}$ which is $\sigma\left(\mathfrak{M}, \mathfrak{M}_{*}\right)$ dense in $\mathfrak{M}$.

Let $\left\{\mathfrak{M}_{\alpha}\right\}_{\alpha \in \Gamma}$ be a net of $W^{*}$-algebras in the sense of [12], Definition 2, i.e.

i) to all pairs $\mathfrak{M}_{\alpha}, \mathfrak{M}_{\beta}$ in $\left\{\mathfrak{M}_{\alpha}\right\}_{\alpha \in \Gamma}$ there exists $\mathfrak{M}_{\gamma}$ with the property $\mathfrak{M}_{\alpha} \cup \mathfrak{M}_{\beta} \subseteq \mathfrak{M}_{\gamma}$.

ii) Every $\mathfrak{M}_{\alpha}$ contains the unit of $\mathfrak{U}$ where $\mathfrak{U}$ is defined in

iii) $\mathfrak{U}=\bar{\bigcup}_{\alpha \in \Gamma} \mathfrak{M}_{\alpha}^{n}((\cdot))^{n}$ is the norm closure of $\left.(\cdot)\right)$.

A $C^{*}$-algebra $\mathfrak{U}$ has a funnel $\left\{\mathfrak{M}_{\alpha}\right\}_{\alpha \in \Gamma}$, whenever $\left\{\mathfrak{M}_{\alpha}\right\}_{\alpha \in \Gamma}$ satisfies i), ii) and iii) above. $\mathfrak{M}_{\alpha}$ is called a component of the funnel. A $C^{*}$-algebra

\footnotetext{
${ }^{1}$ For definitions see $\S 2$.
} 
$\mathfrak{U}$ has a $\sigma$-finite (resp. separable) properly infinite funnel whenever it has a funnel whose components are $\sigma$-finite (respectively separable) properly infinite $W^{*}$-algebras. A $W^{*}$-algebra $\mathfrak{M}$ is separable (i.e. has a separable predual) iff there exists a faithful normal representation of $\mathfrak{M}$ on a separable Hilbert-space. Indeed, let $\pi$ be a faithful normal representation of $\mathfrak{M}$ on a separable Hilbert-space $\mathfrak{H}$. Then $\pi(\mathfrak{M})$ is a von Neumann algebra on $\mathfrak{H}$. Let $\psi \in \pi(\mathfrak{M})_{*}$, then the transposed map ${ }^{t} \pi$ of $\pi$ is defined by $\left({ }^{t} \pi(\psi), A\right)=\psi(\pi(A))$ for all $A \in \mathfrak{M}$. ${ }^{t} \pi\left(\pi(\mathfrak{M})_{*}\right)$ is the image of $\pi(\mathfrak{M})_{*}$ in $\mathfrak{M}_{*}$ because $\pi$ is normal. Furthermore every $\varphi \in \mathfrak{M}_{*}$ is the image of some $\psi \in \pi(\mathfrak{M})_{*}$ under the transpose ${ }^{t} \pi$ of $\pi$. Indeed: $\varphi(A)=\left(\varphi \circ \pi^{-1}\right)(\pi(A))=\left({ }^{t} \pi\left(\varphi \circ \pi^{-1}\right), A\right), A \in \mathfrak{M}$, where $\varphi \circ \pi^{-1}$ is normal because $\varphi$ is normal and $\pi$ is faithful normal. Therefore we have that ${ }^{t} \pi\left(\pi(\mathfrak{M})_{*}\right)=\mathfrak{M}_{*}$. In addition we have that $\left\|^{t} \pi(\psi)\right\|=\|\psi\|$ for all $\psi \in \pi(\mathfrak{M})_{*}$ namely:

$$
\|t \pi(\psi)\|=\sup _{\|A\|=1}\left|\left({ }^{t} \pi(\psi), A\right)\right|=\sup _{\|\pi(A)\|=1}|\psi(\pi(A))|=\|\psi\| .
$$

We thus conclude that $\mathfrak{M}_{*}$ is isometric as a Banach space to $\pi(\mathfrak{M})_{*}$ which in turn is isometric to $B(\mathfrak{H})_{*} / \pi(\mathfrak{M})^{\perp}$, where $B(\mathfrak{H})_{*}$ is the set of trace-class operators on $\mathfrak{H}$ and $\pi(\mathfrak{M})^{\perp}$ is the annihilator of $\pi(\mathfrak{M})$ in $B(\mathfrak{H})_{*}$. Since $\mathfrak{H}$ is separable and $\pi(\mathfrak{M})^{\perp}$ closed, $B(\mathfrak{H})_{*} / \pi(\mathfrak{M})^{\perp}$ is separable and consequently $\pi(\mathfrak{M})_{*}$ (and hence $\mathfrak{M}_{*}$ ) is separable. If we now conversely consider the situation where $\mathfrak{M}_{*}$ is separable, then there exists a countable dense set $\left\{\varphi_{n}\right\}$ in $\mathfrak{M}_{*}^{+}$. From this one constructs $\varphi=\Sigma \frac{1}{2^{n}\left\|\varphi_{n}\right\|} \varphi_{n}$, then $\varphi$ is normal, as a norm-limit of normal linear positive forms, furthermore $\varphi$ is faithful on $\mathfrak{M}$ and gives rise to a faithful normal cyclic representation $\pi_{\varphi}$ of $\mathfrak{M}$ on a separable Hilbert space $\mathfrak{H}_{\varphi}$. The separability of $\mathfrak{H}_{\varphi}$ follows from the fact that the $\sigma\left(\mathfrak{M}, \mathfrak{M}_{*}\right)$ topology on the unit ball of $\mathfrak{M}$ is metrizable and therefore we can find a countable $\sigma\left(\mathfrak{M}, \mathfrak{M}_{*}\right)$ dense subset of $\mathfrak{M}$, $\left\{x_{n}\right\}$ say. Let $\Omega_{\varphi}$ be the cyclic vector for $\pi_{\varphi}(\mathfrak{M})$ in $\mathfrak{H}_{\varphi}$. For every $\xi \in \mathfrak{H}_{\varphi}$ with the property $\left(\xi, \pi_{\varphi}\left(x_{n}\right) \Omega_{\varphi}\right)=0 \forall x_{n} \in \mathfrak{M}$, we have that $\left(\xi, \pi_{\varphi}(x) \Omega_{\varphi}\right)$ $=0 \forall x \in \mathfrak{M}$ because $\omega_{\xi, \Omega_{\varphi}}$ is $\sigma\left(\mathfrak{M}, \mathfrak{M}_{*}\right)$ continuous and $\pi_{\varphi}$ is normal. Because $\Omega_{\varphi}$ is cyclic for $\pi_{\varphi}(\mathfrak{M})$ we conclude that $\xi=0$, and therefore that $\left\{\pi_{\varphi}\left(x_{n}\right) \Omega_{\varphi}\right\}$ is a dense set of vectors in $\mathfrak{H}_{\varphi}$ which then is separable.

A $W^{*}$-algebra $\mathfrak{M}$ is $\sigma$-finite iff there exists a faithful normal state on $\mathfrak{M}$ [8]. From what was said above, it follows that every separable $W^{*}$-algebra is $\sigma$-finite. A particular case is when the algebra $\mathfrak{U}$ could be thought of as generated on Fock space. The funnel then consists of von Neumann algebras on Fock space and because this space is separable every component $\mathfrak{M}_{\alpha}$ in the funnel is also separable and of course $\sigma$-finite.

Let $\mathfrak{U}$ be a $C^{*}$-algebra with a $\sigma$-finite, properly infinite funnel then for every $\mathfrak{M}_{\alpha}$ and $\mathfrak{M}_{\beta}$ with $\mathfrak{M}_{\alpha} \subseteq \mathfrak{M}_{\beta}$ the embedding is normal ([34], Theorem 7). 
A locally normal linear form on $\mathfrak{U}$ is an element from $\mathfrak{U}^{*}$, with the property that its restriction to every $\mathfrak{M}_{\alpha}$ is normal (i.e. $\sigma\left(\mathfrak{M}_{\alpha},\left(\mathfrak{M}_{\alpha}\right)_{*}\right)$ continuous). Because the embeddings $\mathfrak{M}_{\alpha} \subseteq \mathfrak{M}_{\beta}$ for all ordered pairs in the funnel are normal we can apply [12] (Proposition 6) and conclude that the locally normal positive forms on $\mathfrak{U}$ form a folium (i.e. they form a uniformly closed convex subset in $\mathfrak{U}^{*}$, with the property that for every $\varphi$ in this set $\varphi_{A}(\cdot)=\varphi\left(A^{*} \cdot A\right)$ also belongs to it). We shall nowhere assume that we have a full folium.

Let $\mathfrak{U}$ be a $C^{*}$-algebra with a sequential separable funnel. Then in every $\mathfrak{M}_{n}$ in the funnel there exists a separable $C^{*}$-algebra $\mathfrak{U}_{n}$ that is $\sigma\left(\mathfrak{M}_{n},\left(\mathfrak{M}_{n}\right)_{*}\right)$ dense in $\mathfrak{M}_{n}$. Consider the separable $C^{*}$-algebra $\mathfrak{U}_{0}=\bigcup_{n} \mathfrak{\mathfrak { M }}_{n}^{n}$.

Let $V$ denote the set of locally normal linear forms on $\mathfrak{U}$. Let $\mathfrak{S}$ denote the set of states on $\mathfrak{U}$; the set of locally normal states is then $\mathfrak{S}_{\cap} V$. By $\sigma(\mathfrak{S} \cap V, \mathfrak{U})$ we denote the topology induced on $\mathfrak{S} \cap V$ by the $\sigma\left(\mathfrak{U}^{*}, \mathfrak{U}\right)$ topology (i.e. the weak*-topology) on $\mathfrak{U}^{*}$. By $\sigma\left(\mathfrak{S} \cap V, \mathfrak{U}_{0}\right)$ we denote the locally convex topological structure induced on $\varsigma_{\cap} V$ by the semi-norms on $V: \varphi \rightarrow|\varphi(A)|, \varphi \in V, A \in \mathfrak{U}_{0}$. We then obviously have that

$$
\sigma(\mathfrak{S} \cap V, \mathfrak{d}) \prec \sigma\left(\mathfrak{S} \cap V, \mathfrak{H}_{0}\right) .
$$

Let $K$ be any $\sigma(\Im \cap V, \mathfrak{U})$ compact subset of $\subseteq \cap V$. Whenever we can show that $\sigma\left(\mathfrak{S} \cap V, \mathfrak{U}_{0}\right)$ is a Hausdorff topology then $\sigma(\widetilde{S} \cap V, \mathfrak{u})$ and $\sigma\left(\mathfrak{S}_{\cap} V, \mathfrak{U}_{0}\right)$ coincide on $K$ [23]. In that case we have, because $\mathfrak{U}_{0}$ is a separable $C^{*}$-algebra, that $K$ is a metrizable compact in $\sigma(\subseteq \cap V, \mathfrak{U})$. This then is independent of the particular choice of $\mathfrak{U}_{0}$. In order to show that $\sigma\left(\mathfrak{S} \cap V, \mathfrak{U}_{0}\right)$ is a Hausdorff topology it suffices to show that $\sigma\left(V, \mathfrak{U}_{0}\right)$ is Hausdorff because $\sigma\left(\mathfrak{S} \cap V, \mathfrak{U}_{0}\right)$ is the topology induced on $\mathfrak{S} \cap V$ by $\sigma\left(V, \mathfrak{U}_{0}\right)$. Let $\varphi \in V$ and $\varphi \neq 0$, then $\varphi(A)=0 \forall A \in \mathfrak{U}_{0}$ implies $\varphi(x)=0 \forall x \in \mathfrak{M}_{n}, n$ is arbitrary. This is because $\mathfrak{M}_{n}$ is a separable von Neumann algebra and we can find a separable $C^{*}$-algebra $\mathfrak{U}_{n}$ which is $\sigma\left(\mathfrak{M}_{n}, \mathfrak{M}_{n *}\right)$ dense in $\mathfrak{M}_{n}$. Indeed, let $x \in \mathfrak{M}_{n}$ then there exists a sequence $x_{K}^{n}$ in $\mathfrak{U}_{n}$ such that $x_{K}^{n} \rightarrow x \sigma\left(\mathfrak{M}_{n}, \mathfrak{M}_{n *}\right)$ weakly. Since we assumed $\varphi(A)=0 \forall A \in \mathfrak{U}_{0}$ and $\varphi$ is normal (i.e. $\sigma\left(\mathfrak{M}_{n}, \mathfrak{M}_{n *}\right.$ ) continuous) on $\mathfrak{M}_{n}$ we find that $\varphi(x)=0 \forall x \in \mathfrak{M}_{n}, n$ arbitrary. Therefore $\varphi$ vanishes on $\bigcup_{n} \mathfrak{M}_{n}$ and because $\varphi \in \mathfrak{H}^{*}, \varphi$ has to vanish on all of $\mathfrak{U}$, which is in contradiction with the assumption $\varphi \neq 0$. In other words to every $\varphi \in V, \varphi \neq 0$ there exists an element $A \in \mathfrak{U}_{0}$ with the property that $\varphi(A) \neq 0$, implying that $\sigma\left(V, \mathfrak{U}_{0}\right)$ is a Hausdorff topology.

\section{§ 3. K.M.S. States in Connection with Local Normality}

Let $\mathfrak{U}$ be a $C^{*}$-algebra and $t \rightarrow \sigma_{t}$ a representation of the additive group of the real numbers into the group of *automorphisms of $\mathfrak{U}$. 
A state on $\mathfrak{U}$ satisfies the K.M.S. boundary condition, at the inverse temperature $\beta$, whenever the following two conditions hold:

i) $\omega\left(A \alpha_{t} B\right)$ is a continuous function of $t$ for all $A, B \in \mathfrak{U}$.

ii) $\int \omega\left(A \sigma_{t}(B)\right) f(t-i \beta) d t=\int \omega\left(\sigma_{t}(B) A\right) f(t) d t$

for every $f \in \hat{D}$, where $\mathfrak{D}$ is the space of the Fourier-transforms of functions in $\mathfrak{D}$.

[Equivalently $\omega$ satisfies the K.M.S. boundary condition, whenever for each pair $A$ and $B$ in $\mathfrak{U}$ there exists a holomorphic function $F$ in $0<\operatorname{Im} z<\beta$ with boundary values

$$
\left.F(t)=\omega\left(\sigma_{t}(A) B\right) \quad \text { and } \quad F(t+i \beta)=\omega\left(B \sigma_{t}(A)\right) \cdot\right]
$$

For details on the structure of representations induced by a K.M.S. state we refer the reader to $[11,37,40,41]$.

A well known fact by now is that every K.M.S. state $\omega$ as defined above, gives rise, by the Gel'fand-Segal construction, to a representation $\pi_{\omega}$ of $\mathfrak{U}$ with the property that $\pi_{\omega}(\mathfrak{l})^{\prime \prime}$ has a cyclic and separating vector. $\pi_{\omega}(\mathfrak{U})^{\prime \prime}$ is therefore a $\sigma$-finite von Neumann algebra [8].

Theorem 1. Let $\mathfrak{U}$ be a $C^{*}$-algebra with a $\sigma$-finite properly infinite funnel, and $\omega$ a K.M.S. state on $\mathfrak{U}$ then $\omega$ is locally normal.

Proof. Let $\left(\pi_{\omega}, \mathfrak{H}_{\omega}, \Omega_{\omega}\right)$ be the representation, the Hilbert-space and the cyclic vector obtained from the Gel'fand-Segal construction. As already remarked $\Omega_{\omega}$ is separating for $\pi_{\omega}(\mathfrak{l})^{\prime \prime}$ and therefore $\pi_{\omega}(\mathfrak{l})^{\prime \prime}$ is $\sigma$-finite. Let $\mathfrak{M}_{\alpha}$ be a component of the funnel, then $\pi_{\omega} \mid \mathfrak{M}_{\alpha}$ is a ${ }^{*}$-homomorphism of a $\sigma$-finite $W^{*}$-algebra into the $\sigma$-finite algebra $\pi_{\omega}(\mathfrak{l})^{\prime \prime}$. Such a mapping is normal ([34], Theorem 7). Hence $\omega$ is locally normal. q.e.d.

Remark. Since [34] Theorem 7 also holds for every *-homomorphism of a finite factor into a $\sigma$-finite von Neumann-algebra, we see that $\omega$ is normal on every finite factor in $\mathfrak{U}$ and also on every $\sigma$-finite properly infinite $W^{*}$-algebra contained in $\mathfrak{U}$ whether or not contained in the funnel. Of course Theorem 1 holds also if the funnel is a finite-factor-funnel. If we specialize to a sequential separable properly infinite funnel ${ }^{2}$ then we have the following.

Corollary 1. Every K.M.S. state on a $C^{*}$-algebra $\mathfrak{U}$ with a sequential separable properly infinite funnel, is locally normal, acts on a separable Hilbert-space and is normal on every factor contained in $\mathfrak{H}$.

Proof. From Theorem 1 we know that $\pi_{\omega} \mid \mathfrak{M}_{n}$ is normal. $\mathfrak{M}_{n}$ is separable therefore $\left[\pi_{\omega} \mid \mathfrak{M}_{n} \Omega_{\omega}\right]$ is a separable subspace of $\mathfrak{H}_{\omega}$ and thus $\mathfrak{H}_{\omega}\left(=\bigcup_{n}\left(\pi_{\omega} \mid \mathfrak{M}_{n}\right) \Omega_{\omega}\right)$ is separable. By $[10,17,35]$ we know that every representation of a factor on a separable Hilbert-space is normal. q.e.d.

${ }^{2}$ Everywhere in the following the reader can substitute for each properly infinite $W^{*}$-algebra a finite factor without changing the results. 
Corollary 2. For a $C^{*}$-algebra with a sequential separable properly infinite funnel the following statements are equivalent:

i) a state $\omega$ gives rise to a representation on a separable Hilbert-space

ii) $\omega$ is locally normal.

Furthermore whenever i) or ii) holds, $\omega$ is normal on every $\sigma$-finite properly infinite $W^{*}$-algebra and on every factor in $\mathfrak{U}$.

The proof of corollary 2 is a straightforward combination of [34] (Corollary to Theorem 7) and the arguments given in the proofs of Theorem 1 and Corollary $1^{3}$.

Corollary 3. For a $C^{*}$-algebra $\mathfrak{U}$ with a sequential separable properly infinite funnel (respectively a sequential separable factor funnel) the following statements are equivalent:

i) $\omega$ is locally normal

ii) $\omega$ is normal on every $\sigma$-finite properly infinite sub $W^{*}$-algebra of $\mathfrak{U}$ (respectively every subfactor of $\mathfrak{U}$ ).

We want to conclude this section with a few remarks.

Remark 1. Let $\mathfrak{U}$ be a $C^{*}$-algebra $\mathfrak{U}$ with a sequential separable properly infinite funnel $\left\{\mathfrak{M}_{n}\right\}$. Suppose furthermore that $\left\{\mathfrak{M}_{n}\right\}$ is obtained by some sort of "resummation" of a factor funnel $\left\{\mathfrak{F}_{\alpha}\right\}$ where $\alpha$ is an arbitrary index set. The existence of a locally normal state $\omega$ on $\mathfrak{U}$ implies then that every component of $\left\{\mathfrak{F}_{\alpha}\right\}$ is separable. [This is true since $\mathfrak{H}_{\omega}$ is separable and hence $\pi_{\omega} \mid \mathfrak{F}_{\alpha}$ is normal and faithful, because $\mathfrak{F}_{\alpha}$ is a factor].

Remark 2. A $C^{*}$-algebra $\mathfrak{U}$ with a funnel of which at least one component is not a finite $W^{*}$-algebra, does not admit a representation of finite type and in particular it does not admit an infinite temperature K.M.S. state. The latter statement holds because an infinite temperature K.M.S. state gives rise to a finite trace on $\mathfrak{U}$, which does not vanish on the components of the funnel. (Every component contains the unit of $\mathfrak{U}$.)

Remark 3. None of the algebras that admit a central state can have a funnel structure of local $W^{*}$-algebras, with at least one properly infinite component. Not surprisingly, this is the case for the C.A.R. algebra and the algebra of a Quantum-lattice gas. It is also the case for the C.C.R. algebra considered in [22]. The fact that this algebra has a central state has been shown in [38].

Remark 4. Let $\mathfrak{U}$ be the algebra of all bounded operators on an infinite dimensional, separable Hilbert space $\mathfrak{H}$. Let $H$ be a selfadjoint operator on $\mathfrak{H}$ whose spectrum is not totally discrete, with finite multi-

${ }^{3}$ Corollaries 1 and 2 hold also for $C^{*}$-algebras with a sequential separable factor funnel. Corollary 1 for a $C^{*}$-algebra with a sequential separable factor funnel was shown in [12]. 
plicities. Then there exists no K.M.S. state on $\mathfrak{U}$ with respect to the automorphism group:

$$
A \in \mathfrak{U} \mapsto \sigma_{t}(A)=U_{t} A U_{-t}, \quad U_{t}=\exp i H t .
$$

To see this, suppose one had indeed a state $\omega$ that were K.M.S. with respect to $\sigma_{t}$ at a certain value of $\beta$, which we take 1 for simplicity. Then because of Theorem $1 \omega$ has to be normal, i.e. $\omega(A)=\operatorname{Tr}(\varrho A), A \in \mathfrak{A}$.

Thus $\pi_{\omega}$ is faithful. Also $\omega\left(A^{*} A\right)=0$ implies $\pi_{\omega}(A)=0$ and hence $A=0$. The latter fact means that $\varrho$ is invertible i.e. $\varrho=\exp \left(-H^{\prime}\right)$, where $H^{\prime}$ has a totally discrete spectrum with finite multiplicities. From the standard construction $[11,41]$ we know that $\omega$ is also K.M.S. at the temperature 1 , for the automorphism group $\gamma_{t}$ :

$$
A \rightarrow \gamma_{t}(A)=V_{t} A V_{-t}, \quad V_{t}=\exp \left(i H^{\prime} t\right) .
$$

Since $\omega$ is a faithful state on $\mathfrak{U}$ we have then $[32,37]$ that

$$
\sigma_{t}(A)=\gamma_{t}(A) .
$$

This equation implies that $U_{t} V_{-t} \in B(\mathfrak{h})^{\prime}=\{\lambda 1\}$, i.e. $U_{t} V_{-t}=\exp i \alpha(t)$ where $t \rightarrow \alpha(t)$ is a real function on the real line.

Furthermore i) expi $i(t)$ is a continuous function of $t$, ii) $\exp i \alpha\left(t_{1}+t_{2}\right)$ $=\exp i\left[\alpha\left(t_{1}\right)+\alpha\left(t_{2}\right)\right]$.

Applying Stone's theorem in a 1-dimensional space we conclude that $\alpha(t)=\lambda t$ where $\lambda$ is a real constant. This, however, means $H=H^{\prime}+\lambda$, which contradicts our assumption about the spectrum of $H$. q.e.d.

\section{§4. The Simplex Structure of Various Sets of States on a $C^{*}$-Algebra 2}

Our ultimate goal in this chapter is to make statements about various possible decompositions of states on a $C^{*}$-algebra $\mathfrak{U}$. In order to do so we will first make some statements about the lattice structure of various sets of functionals on a $C^{*}$-algebra.

Let $K$ be a convex set of states on $\mathfrak{U}$. Denote by $\hat{K}$ the positive cone generated by $K$, i.e. for every $\mu \in \hat{K}$ we can find $\mu_{0} \in K$ and $\lambda>0$ such that $\mu=\lambda \mu_{0}$. For the moment we do not assume that $K$ is closed in the $\sigma\left(\mathfrak{U}^{*}, \mathfrak{U}\right)$ topology [Since the $\sigma\left(\mathfrak{U}^{*}, \mathfrak{U}\right)$ topology is Hausdorff, closedness of $K$ would imply compactness of $K]$. Consider $\hat{K}-\hat{K}$ endowed with its own order, i.e. for

$$
z_{i} \in \hat{K}-\hat{K}(i=1,2) z_{1} \leqq z_{2} \quad \text { means } z_{2}-z_{1} \in \hat{K} .
$$

For $\omega \in \hat{K}$ we define

$$
S(\omega)=\left\{\varrho \in \hat{K}: \exists \lambda_{\varrho}>0 \quad \text { with } \quad \lambda_{\varrho} \omega-\varrho \in \hat{K}\right\} .
$$


Every $\varrho \in S(\omega)$ defines a positive operator $T_{\varrho}$ in $\pi_{\omega}(\mathfrak{l})^{\prime}$ with the property that:

$$
\varrho(A)=\left(\Omega_{\omega}, T_{\varrho} \pi_{\omega}(A) \Omega_{\omega}\right)=\omega_{T_{e}^{\frac{1}{2}} \Omega_{\omega}}(A), \quad A \in \mathfrak{U} .
$$

Define for every $\omega \in \hat{K}, C_{S(\omega)}$ by:

$$
C_{S(\omega)}=\left\{T_{\varrho} \in\left(\pi_{\omega}(\mathfrak{u})^{\prime}\right)^{+}: \omega_{T_{e}^{\frac{1}{2}} \Omega_{\omega}} \in S(\omega)\right\} .
$$

The map $\varrho \in S(\omega) \rightarrow T_{\varrho} \in C_{S(\omega)}$ is order preserving and one-to-one onto if we endow $C_{S(\omega)}$ with its own order, i.e. $T_{\varrho_{1}} \geqq T_{\varrho_{2}}$ means $T_{\varrho_{1}}-T_{\varrho_{2}} \in C_{S(\omega)}$ and $S(\omega)$ with the order induced by $\hat{K}$. The order on $S(\omega)$ induced by $\hat{K}$ coincides, however, with $S(\omega)$ 's own order. We shall be interested in the lattice properties of $\hat{K}$ in its own order (or equivalently in the lattice properties of $\hat{K}-\hat{K}$ when its positive cone is taken to be $\hat{K}$ ). One easily proves the following:

Theorem 4.1. The following statements are equivalent:

i) $\hat{K}$ is a lattice in its own order

ii) for every $\omega \in \hat{K}, S(\omega)$ is a lattice in its own order

iii) for every $\omega \in \hat{K}, C_{S(\omega)}$ is a lattice in its own order.

Suppose that $\hat{K}$ is a lattice in its own order. Then from the fact that we have a one-to-one order preserving map

$$
C_{S(\omega)} \leftrightarrow S(\omega)
$$

we cannot expect that $C_{S(\omega)}$ is the positive portion of an abelian algebra. It is just a lattice isomorphism. To illustrate this remark let us consider the following:

Let $\mathfrak{A}$ be the $C^{*}$-algebra of $3 \times 3$ matrices and let

$$
a=\left(\begin{array}{lll}
1 & 1 & 0 \\
1 & 1 & 0 \\
0 & 0 & 1
\end{array}\right), \quad b=\left(\begin{array}{rrr}
1 & -1 & 0 \\
-1 & 1 & 0 \\
0 & 0 & 0
\end{array}\right) .
$$

Let $V$ be the two-dimensional subspace of $\mathfrak{A}$ consisting of all $\alpha a+\beta b$; $\alpha, \beta \in \mathbb{C}$. Making use of the trace we can consider $\mathfrak{A}$ itself as the conjugate space of $\mathfrak{A}$, because $\mathfrak{A}$ is finite dimensional. Let $K$ be the intersection of $V$ with the state space $S$ of $\mathfrak{A}$, i.e.

$$
K=\left\{\omega \in S: \omega(x)=\operatorname{Tr}(\alpha \times a+\beta \times b), x \in \mathfrak{A}, \text { for } \alpha, \beta \in R^{+}\right\} .
$$

Since the functional: $x \in \mathfrak{A} \mapsto \operatorname{Tr} x y \in \mathbb{C}$ is positive iff $y$ is positive as an operator, $\omega_{\alpha, \beta}(x)=\operatorname{Tr}[x(\alpha a+\beta b)]$ is positive iff $\alpha a+\beta b$ is positive; this is only true if $\alpha \geqq 0$ and $\beta \geqq 0$. Therefore, the self-adjoint portion of $V$, which is the real-linear span of $a$ and $b$, is a lattice the order being the natural order on $V$ derived from the natural order on positive operators. 
But we can easily see that $a \cdot b \notin V$. (This example is adapted from the example in [31].)

Suppose $K$ were a compact convex set in $\mathfrak{U}^{*}$, then the equivalent statements of Theorem 4.1 would mean that $K$ is a Choquet-simplex.

Before discussing examples of sets of positive linear functionals that satisfy one of the equivalent conditions of Theorem 4.1 we need some lemma's and definitions.

Let $\mathfrak{U}$ be a $C^{*}$-algebra, and let $G$ be a group that is represented as a group of *automorphisms of $\mathfrak{U}$. Let $I_{G}(\mathfrak{U})$ denote the set of invariant states under the action of $G$, i.e. $I_{G}(\mathfrak{l})=\left\{\omega \in S(\mathfrak{U}): \omega\left(\alpha_{g}(A)\right)=\omega\right\}$.

Suppose that $I_{G}(\mathfrak{l}) \neq \emptyset$ (the empty set).

Define for $\omega \in I_{G}(\mathfrak{U})$ on $\mathfrak{H}_{\omega}$ the projection operator $P_{0}^{\omega}$ as the projection operator on $\left\{\psi \in \mathfrak{H}_{\omega}: U_{g}^{\omega} \psi=\psi\right\}$.

We have then the following:

Lemma 4.2. $\mathfrak{U}$ is G-abelian iff $P_{0}^{\omega}$ is an abelian projection in

$$
\left[\pi_{\omega}(\mathfrak{U}) \cup U_{g}^{(\omega)}\right]^{\prime \prime} \quad \text { for all } \omega \in I_{G}(\mathfrak{U}) \text {. }
$$

Proof. As one easily verifies

$$
\left[\pi_{\omega}(\mathfrak{U}) \cup P_{0}^{\omega}\right]_{P_{0}^{\omega}}^{\prime \prime}=\left[P_{0}^{\omega} \pi_{\omega}(\mathfrak{U}) P_{0}^{\omega}\right]_{P_{0}^{\omega}}^{\prime \prime} .
$$

Since by definition [21], $\mathfrak{U}$ is $G$-abelian when for all $\omega \in I_{G}(\mathfrak{l})\left\{P_{0}^{\omega} \pi(\mathfrak{U}) P_{0}^{\omega}\right\}$ is abelian, we conclude that $G$-abelianness of $\mathfrak{U}$ implies abelianness of the right hand side of (4.1) and hence $P_{0}^{\omega}$ is an abelian projection in $\left[\pi_{\omega}(\mathfrak{l}) \cup P_{0}^{\omega}\right]^{\prime \prime}$, but this algebra is the same as $\left[\pi_{\omega}(\mathfrak{l}) \cup U_{g}^{\omega}\right]^{\prime \prime}$. This proves the only if part. To prove the if part suppose that $P_{0}^{\omega}$ is an abelian projection in $\left[\pi_{\omega}(\mathfrak{U}) \cup U_{g}^{\omega}\right]^{\prime \prime}$ then it is also an abelian projection in $\left[\pi_{\omega}(\mathfrak{U}) \cup P_{0}^{\omega}\right]^{\prime \prime}$. Furthermore since $\left[P_{0}^{\omega} \pi_{\omega}(\mathfrak{l}) P_{0}^{\omega}\right]_{P_{0}^{\omega}}^{\prime \prime}$ is *-isomorphic to $P_{0}^{\omega}\left[P_{0}^{\omega} \pi(\mathfrak{H}) P_{0}^{\omega}\right]_{P_{0}^{\omega}}^{\prime \prime}$ we see that $\left[P_{0}^{\omega} \pi_{\omega}(\mathfrak{U}) P_{0}^{\omega}\right]$ is an abelian set of operators and hence $\mathfrak{U}$ is $G$-abelian. $\quad$ q.e.d.

Definition 4.3. $\mathfrak{U}$ is $G^{\prime}$-abelian when for every $\omega \in I_{G}(\mathfrak{l})$ the projection on $\overline{\left[\pi_{\omega}(\mathfrak{U}) \cup U_{g}^{\omega}\right]^{\prime} \Omega_{\omega}}$ is an abelian projection in $\left[\pi_{\omega}(\mathfrak{l}) \cup U_{g}^{\omega}\right]^{\prime \prime}$ (or equivalently for every $\omega \in I_{G}(\mathfrak{l}),\left[\pi_{\omega}(\mathfrak{l}) \cup U_{g}^{\omega}\right]^{\prime}$ is abelian).

Lemma 4.4. $\mathfrak{U}$ is $G$-abelian implies:

i) $\mathfrak{U}$ is $G^{\prime}$-abelian,

ii) $P_{0}^{\omega}=P^{\omega}$ (where $P^{\omega}$ projects onto $\overline{\left[\pi_{\omega}(\mathfrak{l}) \cup U_{g}^{\omega}\right]^{\prime} \Omega_{\omega}}$ ).

Proof. i) is immediate from the proof of the previous lemma, if we observe that $\left[\pi_{\omega}(\mathfrak{U}) \cup P_{0}^{\omega}\right]_{P \omega}^{\prime \prime}$ as an abelian algebra on $P_{0}^{\omega} \mathfrak{H}$ has a cyclic vector, namely $\Omega_{\omega}$ and hence

$$
\left[\pi_{\omega}(\mathfrak{U}) \cup P_{0}^{\omega}\right]_{P_{0}^{\omega}}^{\prime \prime}=\left[\pi_{\omega}(\mathfrak{l}) \cup P_{0}^{\omega}\right]_{P_{0}^{\omega}}^{\prime}=\left[\pi_{\omega}(\mathfrak{U}) \cup U_{g}^{\omega}\right]_{P_{0}^{\omega}}^{\prime} .
$$

Furthermore the abelian von Neumann algebra $\left[\pi_{\omega}(\mathfrak{l}) \cup U_{g}^{(\omega)}\right]^{\prime}$ is *-isomorphic to $\left[\pi_{\omega}(\mathfrak{l}) \cup U_{g}^{\omega}\right]_{P_{o}^{\omega}}^{\prime}$. Hence i) is proven. 
To prove ii) we observe that $P^{\omega} \leqq P_{0}^{\omega}$ and since $P_{0}^{\omega}$ and $P$ both belong to $\left[\pi_{\omega}(\mathfrak{l}) \cup U_{g}^{\omega}\right]^{\prime \prime}$ and $P_{0}^{\omega}$ is abelian we have that $P^{\omega}=P_{0}^{\omega} G$ where $G$ is a projection in $\left[\pi_{\omega}(\mathfrak{l}) \cup U_{g}^{\omega}\right]^{\prime \prime} \cap\left[\pi_{\omega}(\mathfrak{l}) \cup U_{g}^{\omega}\right]^{\prime}$.

We have that $P^{\omega} \Omega_{\omega}=P_{0}^{\omega} G \Omega_{\omega}$ this implies $\Omega_{\omega}=G \Omega_{\omega}$ and hence, because $G \in \pi_{\omega}(\mathfrak{L})^{\prime}, G=1$ implying $P^{\omega}=P_{0}^{\omega}$.

Lemma 4.5. Let $\omega \in I_{G}(\mathfrak{U})$ be separating then $P^{\omega}=P_{0}^{\omega}$. The proof can be found in [15], Lemma 2.2. It is perhaps worthwhile to mention that this lemma holds independently of whether $\mathfrak{U}$ is $G^{\prime}$ (or $G$ ) abelian or not.

We now want to give a few examples of cones that are lattices in their own order.

I) Let as before $I_{G}(\mathfrak{H})$ be the set of invariant states under the action of a group $G$ and let $\mathfrak{U}$ be $G^{\prime}$-abelian. Due to the fact that invariance of a state is linear (i.e. $\varrho_{1}, \varrho_{2}$ invariant implies $\alpha \varrho_{1}+\beta \varrho_{2}$ is invariant) $C_{S(\omega)}$ 's own order is the usual order on self-adjoint operators on $\mathfrak{H}_{\omega}$. Furthermore $G^{\prime}$-abelianess of $\mathfrak{U}$ implies that $C_{S(\omega)}$ is the positive portion of a commutative von Neumann algebra, namely the set of invariant elements in $\pi_{\omega}(\mathfrak{U})^{\prime}$. Therefore $C_{S(\omega)}$ is a lattice in its own order and hence $I_{G}(\mathfrak{U})$ is also a lattice in its own order. Since for an algebra $\mathfrak{U}$ that is $G$-abelian, it follows that $\mathfrak{U}$ is $G^{\prime}$-abelian we recover the fact that $I_{G}(\mathfrak{U})$ is a lattice in its own order for a $G$-abelian algebra.

II) Let $K_{\beta}$ be the set of K.M.S. states a certain temperature $\beta$. Then $K_{\beta}$ is a lattice in its own order because $C_{S(\omega)}$ is the positive portion of the centre of $\pi_{\omega}(\mathfrak{U})^{\prime \prime}$. Indeed for $\omega \in \hat{K}_{\beta}, \varrho \in S(\omega)$ we have not only that $T_{\varrho} \in\left(\pi_{\omega}(\mathfrak{l})^{\prime}\right)^{+}$but also that $T_{\varrho} \in\left(\pi_{\omega}(\mathfrak{H})^{\prime \prime} \cap \pi_{\omega}(\mathfrak{l})^{\prime}\right)^{+}$. This is true for the following reasons:

$\alpha)$ The K.M.S. condition extends to the von Neumann algebra $\pi_{\omega}(\mathfrak{l})^{\prime \prime}$, i.e.

$$
\int\left(\Omega_{\omega}, A U_{t} B \Omega_{\omega}\right) f(t-i \beta) d t=\int\left(\Omega_{\omega}, B U_{-t} A \Omega_{\omega}\right) f(t) d t \quad \hat{f} \in D,
$$

$A, B \in \pi_{\omega}(\mathfrak{U})^{\prime \prime}$.

$\beta$ ) Since $\varrho \in S(\omega)$ we have, independent of the fact that $\omega$ satisfies K.M.S., a positive operator in $\pi_{\omega}(\mathfrak{l})^{\prime}$ such that $\omega_{T_{e}^{\frac{1}{2}} \Omega_{\omega}}=\varrho$. Since $\varrho$ satisfies K.M.S. we have

$$
\int\left(\Omega_{\omega}, T_{\varrho} A U_{t} B \Omega_{\omega}\right) f(t-i \beta) d t=\int\left(\Omega_{\omega}, T_{\varrho} U_{t} B U_{-t} A \Omega_{\omega}\right) f(t) d t .
$$

Let $A=1$ then, by the invariance that follows from the above equation, we have

$$
U_{t} T_{\varrho} \Omega_{\omega}=T_{\varrho} \Omega_{\omega} \Rightarrow U_{t} T_{\varrho} U_{-t}=T_{\varrho} .
$$

$\gamma)$ The involution $J$ that follows from (4.2) has the properties

$$
\begin{aligned}
J T_{\varrho} J & =K_{\varrho} \in \pi_{\omega}(\mathfrak{U})^{\prime \prime} \cap\left(U_{t}\right)^{\prime} . \\
J T_{\varrho} J \Omega_{\omega} & =K_{\varrho} \Omega_{\omega} \rightarrow J T_{\varrho} \Omega_{\omega}=K_{\varrho} \Omega_{\omega} .
\end{aligned}
$$


Since $K_{\varrho}$ is invariant we have $J T_{\varrho} \Omega_{\omega}=T K_{\varrho} \Omega_{\omega}$ where $T=\exp -\beta / 2 H$ $\left(U_{t}=\exp i H t\right)$.

From this we conclude $T_{Q} \Omega_{\omega}=J T K_{Q} \Omega_{\omega}=K_{Q}^{*} \Omega_{\omega}=K_{Q} \Omega_{\omega}$. [From K.M.S. follows that $\left.J T A \Omega_{\omega}=A^{*} \Omega_{\omega} \forall A \in \pi_{\omega}(\mathfrak{l})^{\prime \prime}\right]$. If we now substitute $K_{\varrho} \Omega_{\omega}=T_{\varrho} \Omega_{\omega}$ in the K.M.S. condition for $\varrho$, we have

$$
\int\left(\Omega_{\omega}, K_{\varrho} A U_{t} B \Omega_{\omega}\right) f(t-i \beta) d t=\int\left(\Omega_{\omega}, K_{\varrho} B U_{-t} A \Omega_{\omega}\right) f(t) d t .
$$

The left-hand side equals $\int\left(\Omega_{\omega}, B U_{-t} K_{Q} A \Omega_{\omega}\right) f(t) d t$ because $\omega$ satisfies K.M.S. We have thus obtained:

$$
\int\left(\Omega_{\omega}, B U_{-t} K_{\varrho} A \Omega_{\omega}\right) f(t)=\int\left(\Omega_{\omega}, K_{\varrho} B U_{-t} A \Omega_{\omega}\right) f(t) d t .
$$

This implies

$$
\left(\Omega_{\omega},\left[B, K_{\varrho}\right] A \Omega_{\omega}\right)=0 \quad \forall A, B \in \pi_{\omega}(\mathfrak{U})^{\prime \prime} .
$$

Hence $\left[B, K_{\varrho}\right] \Omega_{\omega}=0 \Rightarrow\left[B, K_{\ell}\right]=0$, because $\Omega_{\omega}$ is separating for $\pi_{\omega}(\mathfrak{l})^{\prime \prime}$. Therefore $K_{\varrho} \in \pi_{\omega}(\mathfrak{U})^{\prime \prime} \cap \pi_{\omega}(\mathfrak{U})^{\prime}$ and since $J T_{\varrho} J=K_{\varrho}$ we have that $T_{\varrho}=J K_{\varrho} J=K_{\varrho}^{*}=K_{\varrho} \in \pi_{\omega}(\mathfrak{l})^{\prime \prime} \cap \pi_{\omega}(\mathfrak{l})^{\prime}$.

Conversely, due to the pointwise invariance of $\pi_{\omega}(\mathfrak{l})^{\prime \prime} \cap \pi_{\omega}(\mathfrak{l} \mathfrak{l})^{\prime}$ under the action of the modular automorphism, every $T_{\varrho} \in \pi_{\omega}(\mathfrak{l})^{\prime \prime} \cap \pi_{\omega}(\mathfrak{l})^{\prime}$ gives rise to a functional $\varrho=\omega_{T_{e}^{\frac{1}{2}} \Omega_{\omega}} \in S(\omega)$.

The reasoning displayed above implies also that $\omega \in$ Ext $K_{\beta}$ is equivalent with $\omega$ is primary.

III) Let $G$ be any group and let $g \in G \rightarrow \tau_{g}$ be a representation of $G$ in the *-automorphisms of $\mathfrak{U}$. Suppose that $I_{G}(\mathfrak{l})$ (i.e. the set of $\tau_{g}$ invariant states) is not the empty set and furthermore that $K_{\beta} \cap I_{G}(\mathfrak{u}) \neq \emptyset$. (The latter fact in the case of a simple algebra implies that $\tau_{g}$ commutes with the K.M.S. automorphisms [32].)

In this case one easily checks that $C_{S(\omega)}$ is the positive portion of the von Neumann algebra of $\tau_{g}$ invariant elements in $\pi_{\omega}(\mathfrak{l} \mathfrak{l})^{\prime \prime} \cap \pi_{\omega}(\mathfrak{l} \mathfrak{l})^{\prime}$ and therefore $K_{\beta} \cap I_{G}(\mathfrak{U})$ is a lattice in its own order.

In example I) we have that $I_{G}(\mathfrak{H})$ is $\sigma\left(\mathfrak{U}^{*}, \mathfrak{U}\right)$ compact and hence $I_{G}(\mathfrak{l})$ is a simplex for a $G^{\prime}$-abelian algebra $\mathfrak{H}$.

In examples II) and III) we will also have simplices provided $K_{\beta}$ is compact.

The set $K_{\beta}$ of K.M.S. states, for a given K.M.S. automorphism group and a given temperature, is a convex weak $*$-compact subset of $\mathfrak{S}$ if $\sigma_{t}$ is a strongly continuous automorphism group (i.e. $\left\|\sigma_{t}(x)-x\right\| \underset{t \rightarrow 0}{\longrightarrow} 0$ ) of $\mathfrak{U}$. A situation like this occurs in the case of a Quantum lattice gas [26] and in the case of the C.A.R. algebra, if the relevant automorphism group is a so-called quasi-free evolution [27]. In both these cases, under suitable conditions, $K_{\beta}$ consists of one point only and is therefore trivially compact; this one K.M.S. state is primary [21, 27, 32]. 
We shall not assume the strong continuity of the automorphism group $\sigma_{t}: \mathfrak{U} \rightarrow \mathfrak{U}$ but rather we shall assume the compactness of $K_{\beta}$ in the weak*-topology. In order to illustrate this assumption let us firstly recall a result from [18]: Let $\mathfrak{M}$ be a $\sigma$-finite $W^{*}$-algebra with no type $I I_{1}$ portion, and let $\sigma_{t}$ be a group of automorphisms of $\mathfrak{M}$ with the property that $\left\|\sigma_{t}(x)-x\right\| \underset{t \rightarrow 0}{\longrightarrow} 0, x \in \mathfrak{M}$. Then $\left\|\sigma_{t}-1\right\| \underset{t \rightarrow 0}{\longrightarrow} 0$, where 1 is the identity automorphism of $\mathfrak{M}$. From this follows [16], that the automorphisms $\sigma_{t}$ are inner automorphisms implemented by a unitary group in $\mathfrak{M}$ with a bounded generator. Let now $\mathfrak{M}$ be a non type $I I_{1}$, $\sigma$-finite factor, $\omega$ a K.M.S. state on $\mathfrak{M}, \sigma_{t}$ the automorphism. Suppose $\left\|\sigma_{t}(x)-x\right\| \underset{t \rightarrow 0}{\longrightarrow} 0$, then from what we said above $\sigma_{t}(x)=U_{t} x U_{t}^{-1}$ $U_{t} \in \mathfrak{M}$. $U_{t}$ has a bounded generator, i.e. $\left\|U_{t}-1\right\| \underset{t \rightarrow 0}{\longrightarrow} 0$. From [37], Theorem 14.1 and the interpretation of [5] and [25] presented in [40], we conclude that $\mathfrak{M}$ has to be semi-finite and consequently there exists a faithful normal semi-finite trace $\tau$ on $\mathfrak{M}$. Furthermore $\omega(x)=\tau(x h)$, where $h$ is a uniquely defined positive operator in $L^{1}(\mathfrak{M}, \tau)$ and furthermore $\sigma_{t}(x)=h^{i t} x h^{-i t}[37]$. Since we assumed that $\mathfrak{M}$ is a factor (for the nonfactor case an analogous reasoning can be given) $h^{i t}=e^{i \alpha t} U_{t}$ where $\alpha$ is a real number and consequently $\left\|h^{i t}-1\right\| \underset{t \rightarrow 0}{\longrightarrow} 0$, i.e. $\log h$ is a bounded operator. In particular $h$ is then bounded, belongs to $\mathfrak{M}$, has a bounded inverse and because $h$ belongs to the defining ideal $\mathfrak{M}_{\tau}$ of $\tau$ we see that the unit in $\mathfrak{M}$ belongs to $\mathfrak{M}_{\tau}$. Hence $\mathfrak{M}$ is a finite factor.

If we now take a $\sigma$-finite, properly infinite factor, then we know from the existence of a faithful normal state $\Phi$ and Tomita's theorem that this state satisfies the K.M.S. condition with respect to the modular automorphism group $\sigma_{t}^{\Phi}$. Furthermore we know [37] that for this automorphism group $\Phi$ is the only K.M.S. state at the appropriate temperature for $\sigma_{t}^{\Phi} . K_{\beta}$ is therefore compact and $\left\|\sigma_{t}^{\Phi}(x)-x\right\| \underset{t \rightarrow 0}{\longrightarrow} 0$, from what we said above. $A$, by no means general, example of this situation is the finite volume K.M.S. state as considered in $[11,41]$. Before embarking on the decomposition of a K.M.S. state we would like to mention a possibility where the K.M.S. automorphism is not strongly continuous and $K_{\beta}$ is not compact either. Let $\mathfrak{M}$ be a $\sigma$-finite properly infinite $W^{*}$-algebra with a non-atomic centre 3 (i.e. 3 does not contain minimal projections). Suppose $\sigma_{t}$ is a K.M.S. automorphism group and $\Phi$ a K.M.S. state with respect to $\sigma_{t}$, then $\Phi$ is of course normal. (We are considering here the trivial funnel $\mathfrak{M}=\mathfrak{M}_{\alpha}=\mathfrak{U}$.) Whatever topology we equip it with, $K_{\beta}$ will never be compact. The reason being that, although $x \rightarrow \sigma_{t}(x)$ is $\sigma$-strongly continuous if $x \in S(\Phi) \mathfrak{M}(s(\Phi)$ is the support projection of $\Phi)$, every extremal K.M.S. state $\Phi$ has to primary, i.e. $s(\Phi)$ has to be a minimal projection in 3 , which is excluded by assumption. 
As remarked at the beginning of this paragraph our ultimate goal is to exhibit unique decompositions of a state $\omega$ into others which still have properties the original state had.

The "cleanest" decompositions of a state $\omega \in K$, where $K$ is the base of a cone $\hat{K}$ which is a lattice in its own order, are obtainable when $K$ is a compact subset of $S(\mathfrak{H})$ (i.e. when $K$ is a Choquet simplex) and the set of extreme points of $K$ is nice enough.

Indeed if $K$ is a Choquet simplex and Ext $K$ is a Baire set, an $F_{\sigma}$ or a $K$-Borel set one obtains a clean decomposition theorem $[2,24]$. (Given a Choquet simplex this does not exhaust all possibilities of obtaining a unique decomposition; we shall, however, not insist on this any further.)

From now on, we shall assume that $K_{\beta}$ is a $\sigma\left(\mathfrak{U}^{*}, \mathfrak{U}\right)$ compact subset of $S(\mathfrak{U})$, and as remarked before in all three cases (I, II and III) we have a Choquet simplex. Furthermore the set $C_{S(\omega)}$ is the positive portion of a commutative von Neumann algebra in $\pi_{\omega}(\mathfrak{H})^{\prime}$ in all three cases, which we denote by $\mathfrak{B}$, the order on $C_{S(\omega)}$ which makes it a lattice in the usual order on positive operators on $\mathfrak{H}_{\omega}$.

From the fact that $K$ is a Choquet simplex we know that there is a unique maximal measure $\mu_{\omega}$ on $K$ that represents $\omega$ [2]. To exhibit this unique maximal measure, one "diagonalizes" the appropriate, depending on the case at hand, commutative subalgebra $\mathfrak{B}$ in $\pi_{\omega}(\mathfrak{A})^{\prime}$. For completeness we sketch the essential points of such a diagonalization procedure.

Let, for the moment, $\mathfrak{B}$ be an arbitrary commutative von Neumann algebra in $\pi_{\omega}(\mathfrak{U})^{\prime}$ and let furthermore $P=\left[\mathfrak{B} \Omega_{\omega}\right]$. One then observes the following facts:

i) $P \in \mathfrak{B}^{\prime}$ and, therefore, since $[\mathfrak{B}]_{P}$ is a maximal commutative von Neumann algebra on $P \mathfrak{H}_{\omega}$ (it has a cyclic vector!):

$$
\left[\mathfrak{B}^{\prime}\right]_{P}=[\mathfrak{B}]_{P} .
$$

ii) From (4.3) we have that for all $A \in \mathfrak{B}^{\prime}$ there exists a uniquely determined element $\varepsilon(A) \in \mathfrak{B}$ such that

$$
P A P=P \varepsilon(A) .
$$

In particular, since $\pi_{\omega}(\mathfrak{U}) \subset \pi_{\omega}(\mathfrak{H})^{\prime \prime} \subset \mathfrak{B}^{\prime}$, we have

$$
P \pi_{\omega}(A) P=P \varepsilon \circ \pi_{\omega}(A) \quad A \in \mathfrak{U} .
$$

iii) $\left(\Omega_{\omega}, P \pi_{\omega}(A) P \Omega_{\omega}\right)=\left(\Omega_{\omega}, P \varepsilon \circ \pi_{\omega}(A) \Omega_{\omega}\right)=\left(\Omega_{\omega}, \varepsilon^{\circ} \pi_{\omega}(A) \Omega_{\omega}\right)$.

iv) $[\mathfrak{B}]_{P}$ is *-isomorphic to $\mathfrak{B}$ and therefore we have for all $C \in \mathfrak{B}$ :

$$
\left(\Omega_{\omega}, C \Omega_{\omega}\right)=\int_{\Gamma} C(\gamma) d v_{\omega}(\gamma)
$$


where $\Gamma$ is the spectrum space of $\mathfrak{B}$ and $C \rightarrow C(\cdot)$ is the Gel'fand isomorphism of $\mathfrak{B}$ onto $C(\Gamma)$ (the set of continuous functions on $\Gamma$ ). $v_{\omega}$ is the spectral measure on $\Gamma$ determined by $\Omega_{\omega}$. Since $\mathfrak{B}$ is a von Neumann algebra, $\Gamma$ is hyperstonean and [8]:

$$
C(\Gamma) \cong L^{\infty}\left(\Gamma, v_{\omega}\right) .
$$

v) Combining iii) and iv) we obtain:

$$
\left(\Omega_{\omega}, \pi_{\omega}(A) \Omega_{\omega}\right)=\int_{\Gamma}\left(\varepsilon \circ \pi_{\omega}(A)\right)(\gamma) d v_{\omega}(\gamma) .
$$

vi) $B y\left(\varepsilon \circ \pi_{\omega}(A)\right)(\gamma) \equiv \omega_{\gamma}(A) A \in \mathfrak{U}$, one defines for every $\gamma \in \Gamma$, a state $\omega_{\gamma}$ on $\mathfrak{U}$.

Indeed $\omega_{\gamma}$ is linear and normalized to one. Positivity follows from the fact that firstly $\pi_{\omega}\left(A^{*} A\right)$ is positive and secondly that $\mathfrak{B}$ is $*$-isomorphic to $P \mathfrak{B}$. Therefore from (4.3) $\varepsilon \circ \pi_{\omega}\left(A^{*} A\right)$ is positive and hence also $\omega_{\gamma}\left(A^{*} A\right) \geqq 0$.

From vi) we have $\omega(A)=\int_{\Gamma} \omega_{\gamma}(A) d v_{\omega}(\gamma) . \Gamma$ is compact and the map $\varrho: \gamma \rightarrow \omega_{\gamma}$ is a continuous map of $\Gamma$ into $S(\mathfrak{U})\left(S(\mathfrak{U})\right.$ with the $w^{*}$-topology). We therefore obtain a measure $\mu_{\omega}$ on $S(\mathfrak{l})$, by transport of structure, when we define $\mu_{\omega}(f)$ for all $f \in C(S(\mathfrak{U}))$ as:

vii) $\mu_{\omega}(f) \equiv \int_{\Gamma}(f \circ \varrho)(\gamma) d v_{\omega}(\gamma)$.

As one easily sees $\mu_{\omega}$ is a probability measure on $S(\mathfrak{U})$ with its support in $\omega(\Gamma)=\left\{\tau \in S(\mathfrak{U}): \tau=\omega_{\gamma}, \gamma \in \Gamma\right\}$. Since $\gamma \in \Gamma \rightarrow \omega_{\gamma} \in \omega(\Gamma)$ is a continuous map of $\Gamma$ into $S(\mathfrak{l})$, and since $\Gamma$ is compact, $\omega(\Gamma)$ is compact. Therefore we obtain:

$$
\mu_{\omega}(f)=\int_{\omega(\Gamma)} f(\varrho) d \mu_{\omega}(\varrho)=\int_{S(\mathfrak{l})} f(\varrho) d \mu_{\omega}(\varrho) \equiv \int_{\Gamma}(f \circ \varrho)(\gamma) d v_{\omega}(\gamma) .
$$

In particular we obtain

and

$$
\hat{A}(\omega)=\omega(A)=\int_{\omega(\Gamma)} \varrho(A) d \mu_{\omega}(\varrho)
$$

$$
\mu_{\omega}\left(\hat{A}_{1} \ldots \hat{A}_{n}\right)=\left(\Omega_{\omega}, P \pi_{\omega}\left(A_{1}\right) P \ldots P \pi_{\omega}\left(A_{n}\right) P \Omega_{\omega}\right) .
$$

From now on $\mathfrak{B}$ is no longer to be an arbitrary von Neumann-algebra in $\pi_{\omega}(\mathfrak{U})^{\prime}$ but $\mathfrak{B}$ is $\left\{\pi_{\omega}(\mathfrak{l}) \cup U_{g}^{\omega}\right\}^{\prime}$ in case I, $\mathfrak{B}$ is $\pi_{\omega}(\mathfrak{l})^{\prime \prime} \cap \pi_{\omega}(\mathfrak{U})^{\prime}$ in case II and $\mathfrak{B}$ is $\pi_{\omega}(\mathfrak{l})^{\prime \prime} \cap \pi_{\omega}(\mathfrak{l})^{\prime} \cap U_{g}^{\omega^{\prime}}$ in case III. In all these cases we have:

vii) All elements from $\omega(\Gamma)$, i.e. all states $\omega_{\gamma}$, belong to $K$.

This follows from the following reasoning. $\mathfrak{B}$ is a von Neumann algebra hence the unit ball of its predual $\mathfrak{B}_{*}$ is $\sigma\left(\mathfrak{B}^{*}, \mathfrak{B}\right)$ dense in $S(\mathfrak{B})$. This implies that for $C \in \mathfrak{B}$ there exists a net $\left\{\phi_{d}\right\}$ in $\mathfrak{B}_{*}$ with the property

$$
\tilde{\omega}_{\gamma}(C)=\lim _{d} \varphi_{d}(C), \quad \text { where } \quad \tilde{\omega}_{\gamma}(C)=C(\gamma) .
$$


If we now choose $C=\varepsilon \circ \pi_{\omega}(A)$ we have:

$$
\omega_{\gamma}(A)=\left(\varepsilon \circ \pi_{\omega}(A)\right)(\gamma)=\lim _{d} \varphi_{d}\left(\varepsilon \circ \pi_{\omega}(A)\right)
$$

$\varphi_{d}$ as an element of $\mathfrak{B}_{*}$ is a normal state on $\mathfrak{B}$; it is also normal on $[\mathfrak{B}]_{P}$ and this algebra has a cyclic and separating vector in $P \mathfrak{H}_{\omega}$ and hence [8] there exists $a_{d} \in \mathfrak{B}$ such that we may choose

$$
\varphi_{d}\left(\varepsilon \circ \pi_{\omega}(A)\right)=\left(\Omega_{\omega}, a_{d}^{*} \varepsilon \circ \pi_{\omega}(A) a_{d} \Omega_{\omega}\right)
$$

Therefore we have:

$$
\begin{aligned}
\omega_{\gamma}(A) & =\lim _{d}\left(a_{d} \Omega_{\omega}, \varepsilon \circ \pi_{\omega}(A) a_{d} \Omega_{\omega}\right) \\
& =\lim _{d}\left(a_{d} \Omega_{\omega}, \pi_{\omega}(A) a_{d} \Omega_{\omega}\right) .
\end{aligned}
$$

Due to the special choice of the algebra $\mathfrak{B}$ we conclude that $\omega_{\gamma}$ is a pointwise limit of states in $K$. At this point the compactness assumption on $K_{\beta}$ comes into play. Indeed from the compactness of $K$ (in all three cases) it follows that $\omega_{\gamma} \in K$ or equivalently $\omega(\Gamma) \subset K . \mu_{\omega}$ is an $\omega$-representing measure on $K$. Recall that:

$$
\mu_{\omega}\left(\hat{A}_{1} \ldots \hat{A}_{n}\right)=\left(\Omega_{\omega}, P \pi_{\omega}\left(A_{1}\right) \ldots P \pi_{\omega}\left(A_{n}\right) P \Omega_{\omega}\right) .
$$

where $\hat{A}(\omega)=\omega(A)$.

By exactly the same reasoning as used in [28] in order to prove that

$$
\tau_{\omega}\left(\hat{A_{1}} \ldots \hat{A_{n}}\right)=\left(\Omega_{\omega}, P_{0}^{\omega} \pi_{\omega}\left(A_{1}\right) P_{0}^{\omega} \ldots P_{0}^{\omega} \pi\left(A_{n}\right) P_{0}^{\omega} \Omega_{\omega}\right)
$$

extends to the unique maximal $\omega$-representing measure on $I_{G}(\mathfrak{U})$ for $\mathfrak{U} G$-abelian, one proves here

viii) $\mu_{\omega}$ is the unique $\omega$-representing measure that follows from the fact that $K$ is a simplex.

If instead of a $G^{\prime}$-abelian algebra we were to have a $G$-abelian $\mathfrak{U}$ then, since $P_{0}^{\omega}=P^{\omega}$ (Lemma 4.4), we would get that $\mu_{\omega}=\tau_{\omega}$ for case I.

It should be noted that everything we have said up to now holds for arbitrary $C^{*}$-algebras $\mathfrak{U}$, in particular for those which are not separable in the norm-topology. We are now going to specialize to a $C^{*}$-algebra with a sequential separable properly infinite funnel (cf. § 2) and for $K$ we consider from now on only cases II and III. (Such algebras are still non-separable in general.)

Firstly, as we have shown in $\S 2 K_{\beta}$ is a metrizable compact. Therefore its extreme points form a $G_{\delta}$ [2] (hence a Borel set and since $K$ is separable it is a Baire set). The same is true in case III for $K_{\beta} \cap I_{G}(\mathfrak{l})$, where $G$ can be any group represented as *-automorphisms of $\mathfrak{U}$, with the properties that $I_{G}(\mathfrak{U}) \cap K_{\beta} \neq \emptyset$. Therefore we have the following theorems. 
Theorem 4.5. Every K.M.S. state $\omega$ on a $C^{*}$-algebra $\mathfrak{U}$ with a sequential separable properly infinite funnel ${ }^{4}$ can be uniquely decomposed into extremal K.M.S. states (i.e. K.M.S. primary states).

Theorem 4.6. Every K.M.S. state that is also invariant for a *-automorphism group $g \in G \rightarrow \alpha_{g}$ on a $C^{*}$-algebra with a sequential separable properly infinite funnel ${ }^{4}$ can be uniquely decomposed into elements that are extremal elements in $K_{\beta} \cap I_{G}(\mathfrak{U})$.

At this point we want to make two remarks, namely:

1) Given an arbitrary $C^{*}$-algebra and a state $\omega$ thereupon, choose an arbitrary commutative von Neumann algebra $\mathfrak{B}$ in $\pi_{\omega}(\mathfrak{U})^{\prime}$. Then the measure $\mu_{\omega}$ satisfying (4.4) and (4.5) is unique [30]. This, however, does not guarantee that $\mu_{\omega}$ is carried by the extremal points of $K$. It is the simplex structure with additional "niceness" criteria on the set of extremal points that provides the possibility that $\mu_{\omega}$ is carried by the extremal points.

2) Both Theorems 4.5 and 4.6 deal with decompositions of a locally normal state into locally normal states. In $\S 5$ we shall investigate under what conditions a locally normal state can always be decomposed into locally normal states.

We want to conclude this paragraph by describing the additional structure that is obtained when the algebra $\mathfrak{U}$ has somewhat more structure with respect to its *-automorphism group $\left\{\alpha_{g}, g \in G\right\}$.

Suppose that for a certain value of $\beta$ one has that for all $\omega \in K_{\beta} \cap I_{G}(\mathfrak{l})$ :

$$
\pi_{\omega}(\mathfrak{l})^{\prime} \cap U_{g}^{\omega^{\prime}} \subset \pi_{\omega}(\mathfrak{l})^{\prime \prime} \cap \pi_{\omega}(\mathfrak{l})^{\prime} .
$$

Then we have the following

\section{Lemma 4.7. $\quad \operatorname{Ext}\left(K_{\beta} \cap I_{G}(\mathfrak{U})\right) \subset \operatorname{Ext}\left(I_{G}(\mathfrak{U})\right)$.}

Proof. Firstly recall that we assumed that $K_{\beta}$ was compact. Hence $K_{\beta} \cap I_{G}(\mathfrak{u})$ is compact and has extreme points by the Krein-Milman theorem. Let $\omega \in K_{\beta} \cap I_{G}(\mathfrak{U})$ be decomposed as $\omega=\lambda \omega_{1}+(1-\lambda) \omega_{2}$ with $\omega_{i}(i=1,2) \in I_{G}(\mathfrak{U})$. Then $\omega_{i}=\left(T_{i} \Omega_{\omega}, \pi_{\omega}(\cdot) \Omega_{\omega}\right)$ with $T_{i} \in \pi_{\omega}(\mathfrak{l})^{\prime} \cap U_{g}^{\omega^{\prime}}$. By assumption $T_{i} \in \pi_{\omega}(\mathfrak{U})^{\prime \prime} \cap \pi_{\omega}(\mathfrak{H})^{\prime}$ and hence $\omega_{i} \in K_{\beta}$. q.e.d.

We will adopt the definition of a face as given in [9], namely: a face of a convex set $K$ in a vector space is a convex subset $Q$ such that if $\lambda \omega_{1}+(1-\lambda) \omega_{2} \in Q$ with $\omega_{i}(i=1,2)$ and $0<\lambda<1$ then $\omega_{i}(i=1,2) \in Q$. It is then clear from the proof of Lemma 4.7 that we have:

Lemma 4.7. $K_{\beta} \cap I_{G}(\mathfrak{U})$ is a face of $I_{G}(\mathfrak{U})$ (provided $K_{\beta} \cap I_{G}(\mathfrak{U}) \neq \emptyset$ ) iff (4.6) holds for all $\omega \in K_{\beta} \cap I_{G}(\mathfrak{U})$.

\footnotetext{
${ }^{4}$ or a sequential separable factor funnel.
} 
(4.6) is satisfied for $\{\mathfrak{U}, G\}$ asymptotically abelian ([19] and [33], Theorem 5.2) and in the case where $\mathfrak{U}$ is $\mathfrak{M}$ abelian with respect to $\left\{\tau_{g}, g \in G\right\}$ [29]. (In this case $G$ has of course to be amenable.)

Furthermore from the observation i) to vii) we have

Theorem 4.8. Let $\mathfrak{U}$ be a $C^{*}$-algebra admitting a group of K.M.S.automorphisms at a temperature $\beta \neq 0$, with in addition a group of automorphisms $g \in G \rightarrow \alpha_{g}$ such that:

1) $K_{\beta}$ is compact.

2) $K_{\beta} \cap I_{G}(\mathfrak{u}) \neq \emptyset$.

3) $\omega \in K_{\beta} \cap I_{G}(\mathfrak{l}) \rightarrow \pi_{\omega}(\mathfrak{l})^{\prime} \cap U_{g}^{\omega^{\prime}} \subset \pi_{\omega}(\mathfrak{l})^{\prime \prime} \cap \pi_{\omega}(\mathfrak{l})^{\prime}$.

4) $I_{G}(\mathfrak{U})$ is a simplex.

Then the unique maximal measure that represents $\omega$ as an element of the simplex $K_{\beta} \cap I_{G}(\mathfrak{l})$ coincides with the unique maximal measure that represents $\omega$ as an element of the simplex $I_{G}(\mathfrak{l})$.

Corollary 4.9. Let in addition to the assumptions of Theorem 4.8, $\mathfrak{U}$ be a $C^{*}$-algebra with a sequential separable properly infinite funnel (or a sequential separable factor funnel) and $\operatorname{Ext}_{G}(\mathfrak{l})$ be a $\mu_{\omega}$-measurable set. Then the decomposition of $\omega \in K_{\beta} \cap I_{G}(\mathfrak{U})$ into elements of $\operatorname{Ext}\left(K_{\beta} \cap I_{G}(\mathfrak{U})\right)$ coincides with the decomposition of $\omega \in K_{\beta} \cap I_{G}(\mathfrak{U})$ into elements of $\operatorname{Ext}\left(I_{G}(\mathfrak{U})\right)$ or stated differently:

$$
\mu_{\omega}\left(\operatorname{Ext}\left(K_{\beta} \cap I_{G}(\mathfrak{l})\right)\right)=\mu_{\omega}\left(\operatorname{Ext}_{G}(\mathfrak{U})\right)=1, \omega \in K_{\beta} \cap I_{G}(\mathfrak{U}) .
$$

The conditions of Corollary 4.9 are fulfilled for all $\beta \neq 0$ in the case of a lattice gas with the interactions given in [26]; the role of $G$ is played by space-translations, which act asymptotically abelian on $\mathfrak{U}$. Therefore 3 ) and 4) of Theorem 4.8 hold; furthermore $\left\|\sigma_{t}(x)-x\right\| \underset{t \rightarrow 0}{\longrightarrow} 0$ implying that $K_{\beta}$ is compact; $K_{\beta} \cap I_{G}(\mathfrak{U}) \neq \emptyset$. Since $\mathfrak{U}$ is a U.H.F. algebra it is a $C^{*}$-algebra with a sequential separable factor funnel; furthermore $\mathfrak{U}$ is separable in its norm topology and hence Ext $I_{G}(\mathfrak{U})$ is $\mu_{\omega}$-measurable.

\section{§ 5. Decompositions of Locally Normal States into Locally Normal States}

In this paragraph we shall restrict ourselves to $C^{*}$-algebras with a sequential separable factor funnel. This restriction, as we shall see, is essential to the proof of the main theorem of this section; namely

Theorem 5.1. Let $\mathfrak{M}$ be a factor on a separable Hilbert space. Let $\mathfrak{B}$ be a commutative Von Neumann algebra in $\mathfrak{M}^{\prime}$, and let $\Omega$ be a vector in $\mathfrak{H}$ that is separating for $\mathfrak{B}$ (for instance $\Omega$ could be cyclic for $\mathfrak{M}$ ) and

$$
\omega_{\Omega}(A)=(\Omega, A \Omega)=\int_{\Gamma} d \mu(\gamma) \omega_{\gamma}(A) A \in \mathfrak{M},
$$

where $\Gamma, \mu$ and $\omega_{\gamma}$ are as before (cf. $\left.\S 4 \mathrm{i}\right)$ to viii)). 
Then the following statements are equivalent

i) $\omega_{\gamma}$ is $\mu$-a.e. normal on $\mathfrak{M}$.

ii) The natural *-isomorphism

$$
\sum_{i=1}^{n} x_{i} b_{i} \mapsto \sum_{i=1}^{n} x_{i} \otimes b_{i} \quad x_{i} \in \mathfrak{M}, b_{i} \in \mathfrak{B}
$$

extends to a *-isomorphism of

$$
(\mathfrak{M} \cup \mathfrak{B})^{\prime \prime} \quad \text { onto } \mathfrak{M} \bar{\otimes} \mathfrak{B},
$$

where $\mathfrak{M} \bar{\otimes} \mathfrak{B}$ is the tensor product of the Von Neumann algebras $\mathfrak{M}$ and $\mathfrak{B}$.

Proof. Since $\mathfrak{M}$ acts on a separable Hilbert-space, $\mathfrak{M}_{*}$ is separable in its norm topology. It is known that under this condition the predual $(\mathfrak{M} \otimes \mathfrak{B})_{*}$ of $\mathfrak{M} \bar{\otimes} \mathfrak{B}$ is isometric to the Banach space $L^{1}\left(\Gamma, \mathfrak{M}_{*}, \mu\right)$ of all Bochner integrable $\mathfrak{M}_{*}$-valued functions on $\Gamma$ with respect to $\mu$ (see for example [30]).

Suppose (i) holds. Since $\mathfrak{M}$ is a factor and $\mathfrak{B}$ is in $\mathfrak{M}^{\prime}$, the bilinear map: $(x, b) \in \mathfrak{M} \times \mathfrak{B} \rightarrow x b \in B(\mathfrak{S})$ is extended to an isomorphism $\pi_{0}$ of the algebraic tensor product $\mathfrak{M} \otimes \mathfrak{B}$ onto the algebra $R_{0}(\mathfrak{M}, \mathfrak{B})$ generated algebraically by $\mathfrak{M}$ and $\mathfrak{B}$, (see, for example, [8] Chapter I, $\S 2$, Excercise 6). Furthermore, since $\mathfrak{B}$ is abelian, [36] the map $\pi_{0}$ is extended to an isomorphism $\pi$ of the tensor product of $C^{*}$-algebras $\mathfrak{M} \hat{\otimes}_{\alpha} \mathfrak{B}$, onto the $C^{*}$-algebra $\mathfrak{R}(\mathfrak{M} \cup \mathfrak{B})$ generated by $\mathfrak{M}$ and $\mathfrak{B}$. Let $\omega$ be the state on $\mathfrak{R}(\mathfrak{M} \cup \mathfrak{B})$ defined by $\omega(x)=(x \Omega \mid \Omega)$. Put $\tilde{\omega}={ }^{t} \pi(\omega)$. Then $\tilde{\omega}$ is a state of $\mathfrak{M} \hat{\otimes}_{\alpha} \mathfrak{B}$. We claim that $\tilde{\omega}$ is $\sigma$-weakly continuous on $\mathfrak{M} \hat{\otimes}_{\alpha} \mathfrak{B}$ when $\mathfrak{M} \hat{\otimes}_{\alpha} \mathfrak{B}$ is imbedded in the tensor product of von Neumann algebras $\mathfrak{M} \bar{\otimes} \mathfrak{B}$.

$$
\begin{aligned}
& \text { By assumption, we have, for each } \sum_{i=1}^{n} x_{i} \otimes b_{i} \in \mathfrak{M} \otimes \mathfrak{B}, \\
& \qquad \tilde{\omega}\left(\sum_{i=1}^{n} x_{i} \otimes b_{i}\right)=\omega\left(\sum_{i=1}^{n} x_{i} b_{i}\right)=\sum_{i=1}^{n} \int_{\Gamma} \omega_{\gamma}\left(x_{i}\right) b_{i}(\gamma) d \mu(\gamma) .
\end{aligned}
$$

Therefore, $\tilde{\omega}$ is nothing but the restriction of the normal state of $\mathfrak{M} \bar{\otimes} \mathfrak{B}$ which is defined by the $\mathfrak{M}_{*}$-valued function in $L^{1}\left(\Gamma, \mu, \mathfrak{M}_{*}\right): \gamma \in \Gamma \rightarrow \omega_{\gamma}$, to $\mathfrak{M} \hat{\otimes}_{\alpha} \mathfrak{B}$. Thus $\tilde{\omega}$ is $\sigma$-weakly continuous. Since the map $\pi$ is nothing but the cyclic representation of $\mathfrak{M} \hat{\otimes}_{\alpha} \mathfrak{B}$ induced by $\tilde{\omega}, \pi$ is extended to a representation $\tilde{\pi}$ of $\mathfrak{M} \bar{\otimes} \mathfrak{B}$ with the range $(\mathfrak{M} \cup \mathfrak{B})^{\prime \prime}$. Since the centre of $\mathfrak{M} \bar{\otimes} \mathfrak{B}$ is $1 \otimes \mathfrak{B}$ and $\tilde{\pi}$ is faithful on $1 \otimes \mathfrak{B}$ because it is just the identity map, $\tilde{\pi}$ is an isomorphism of $\mathfrak{M} \bar{\otimes} \mathfrak{B}$ onto $(\mathfrak{M} \cup \mathfrak{B})^{\prime \prime}$.

Suppose (ii) holds. Let $\pi$ be the isomorphism of $\mathfrak{M} \otimes \mathfrak{B}$ onto $(\mathfrak{M} \cup \mathfrak{B})^{\prime \prime}$ which extends the map: $(x, b) \in \mathfrak{M} \times \mathfrak{B} \mapsto x b \in R_{0}(\mathfrak{M}, \mathfrak{B})$. Let $\tilde{\omega}$ be the state of $\mathfrak{M} \bar{\otimes} \mathfrak{B}$ defined by $(\pi(x) \Omega \mid \Omega)$. Then $\tilde{\omega}$ belongs to $(\mathfrak{M} \bar{\otimes} \mathfrak{B})_{*}$. 
As we remarked above, there exists a Bochner integrable $\mathfrak{M}_{*}$-valued function: $\gamma \in \Gamma \rightarrow \tilde{\omega}_{\gamma} \in \mathfrak{M}_{*}$ such that

$$
\tilde{\omega}\left(\sum_{i=1}^{n} x_{i} \otimes b_{i}\right)=\sum_{i=1}^{n} \int_{\Gamma} \tilde{\omega}_{\gamma}\left(x_{i}\right) b_{i}(\gamma) d \mu(\gamma)
$$

for each $\sum_{i=1}^{n} x_{i} \otimes b_{i} \in \mathfrak{M} \otimes \mathfrak{B}$. Therefore we have, for each $x \in \mathfrak{M}$,

$$
\begin{aligned}
\omega(x) & =\tilde{\omega}(x \otimes 1) \\
& =\int_{\Gamma} \tilde{\omega}_{\gamma}(x) d \mu(\gamma) .
\end{aligned}
$$

By equality $(*)$ one concludes that the diagonal algebra for the decomposition $(* *)$ of $\omega$ is precisely $\mathfrak{B}$.

Indeed the function $\gamma \in \Gamma \rightarrow \tilde{\omega}_{\gamma} \in \mathfrak{M}_{*}$ is a Bochner integrable function on $\Gamma$ (by the very definition of $L^{1}\left(\Gamma, \mu, \mathfrak{M}_{*}\right)$ ) and hence $\tilde{\omega}_{\gamma}$ is a strongly measurable function on $\Gamma[13,30]$. Therefore by ([1] Chapter IV, $\S 5$, Definition 1 and Proposition 10) on every compact subset $K$ of $\Gamma$ we have that $\tilde{\omega}_{\gamma}$ is a strongly continuous function on $K \backslash N$ where $N$ is a subset of $K$ of $\mu$ measure zero.

Since $\Omega$ is separating for $\mathfrak{B}, \mu$ is a normal basic measure on $\Gamma$; its support is all of $\Gamma$ [8]. Therefore [4], there exists an open dense set $G$ in $\Gamma$ with $G=\bigcup_{n} G_{n}$ where $\left\{G_{n}\right\}$ is a countable family of mutually disjoint open and closed subsets of $\Gamma$. Since $G_{n}$ is a closed subset of $\Gamma$, which is Hausdorff, $G_{n}$ is compact. In the hyperstonean space $\Gamma$ a set is of $\mu$-measure zero iff it is nowhere dense. Furthermore each set of first category is nowhere dense, as is its closure [4].

Returning to $\tilde{\omega}_{\gamma}$, it is clear from what we said above that $\tilde{\omega}_{\gamma}$ is continuous on $G-\bigcup_{n} N_{n}$, with $N_{n} \subset G_{n}$ and $N_{n}$ nowhere dense. Let us denote $G-\widetilde{U N}_{n}$ by $H$. By Baire's theorem $H$ is open dense, indeed $H=G \cap{\overline{\bigcup_{n}}}_{N_{n}}^{c}$ or equivalently $H^{c}=G^{c} \cup \overline{\bigcup_{n} N_{n}}$, i.e. $H_{c}$ is a closed set of first category.

Let now

$$
\omega(A)=\int_{\Gamma} d \mu(\gamma) \omega_{\gamma}(A)
$$

be the decomposition as obtained in $\S 4$ by diagonalizing $\mathfrak{B}$. Then

$$
\omega(A a)=\int_{\Gamma} d \mu(\gamma) \omega_{\gamma}(A) a(\gamma) \quad A \in \mathfrak{M}, a \in \mathfrak{B},
$$

where $\omega_{\gamma}(A)$ is the unique continuous function on $\Gamma$ that coincides, except on a set of measure zero, with $\tilde{\omega}_{\gamma}(A)$ [4]. Define

$$
E(x)=\left\{\gamma \in \Gamma: \tilde{\omega}_{\gamma}(x) \neq \omega_{\gamma}(x)\right\},
$$


then we have that $E(x)$ and hence $\overline{E(x)}$ is nowhere dense. It then follows that $H \cap \overline{E(x)}$ is open dense, and hence

$$
\overline{(H \cap \overline{E(x)}}) \cap H=H .
$$

For $\gamma \in H$, we can find $\gamma_{\alpha}$ such that $\lim _{\alpha} \gamma_{\alpha}=\gamma$ and $\gamma_{\alpha} \in H \cap \overline{E(x)}^{c}$.

Since $\tilde{\omega}_{\gamma_{\alpha}}(x)=\omega_{\gamma_{\alpha}}(x)$ and since $\tilde{\omega}_{\gamma}$ is strongly continuous on $H$, together with the fact that $\omega_{\gamma}$ is continuous on all of $\Gamma$ we have that $\tilde{\omega}_{\gamma}(x)=\omega_{\gamma}(x) \forall x \in \mathfrak{M}, \gamma \in H$. $\quad$ q.e.d.

Let $\mathfrak{U}$ be a sequential separable factor funnel and let $\omega$ be a locally normal state thereupon. It follows from corollary 3 that $\mathfrak{Y}_{\omega}$ is separable. Consider $\overline{\left[\pi_{\omega}\left(\mathfrak{U}_{n}\right) \cup \mathfrak{B}\right] \Omega_{\omega}}=\mathfrak{H}_{\omega}^{n}$ where $\mathfrak{B}$ is a commutative Von Neumann algebra in $\pi_{\omega}(\mathfrak{L})^{\prime}$.

One then has the following structure

a) $\left.\pi_{\omega}\right|_{\mathfrak{U}_{n}}$ is a representation of the factor $\mathfrak{U}_{n}$ on the separable space $\mathfrak{H}_{\omega}$, hence $\left.\pi_{\omega}\right|_{\mathfrak{U}_{n}}$ is faithful normal $[10,17,35]$ and hence $\left.\pi_{\omega}\right|_{\mathfrak{u}_{n}}$ is a Von Neumann algebra; but by faithfulness it is a factor. Let $P_{n} \mathfrak{G}_{\omega}=\mathfrak{H}_{\omega}^{n}$ then $P_{n} \in \pi_{\omega}\left(\mathfrak{U}_{n}\right)^{\prime} \cap \mathfrak{B}^{\prime}$;

$\beta)[\mathfrak{B}]_{P_{n}}$ is isomorphic to $\mathfrak{B} ; \pi_{\omega}\left(\mathfrak{U}_{n}\right)$ is isomorphic to $\left[\pi_{\omega}\left(\mathfrak{U}_{n}\right)\right]_{P_{n}}$ since $\left[\pi_{\omega}\left(\mathfrak{U}_{n}\right)\right]_{P_{n}}$ is the image of a representation of the factor $\pi_{\omega}\left(\mathfrak{U}_{n}\right)$ on $\mathfrak{H}_{\omega}^{n}$ which is separable;

$\gamma)$ In $\mathfrak{S}_{\omega}^{n}$ we have the conditions of Theorem 5.1. Indeed $\Omega$ is separating for $[\mathfrak{B}]_{P_{n}},[\mathfrak{B}]_{P_{n}} \subset\left[\pi_{\omega}(\mathfrak{l})\right]_{P_{n}}^{\prime}$ and $\left[\pi_{\omega}(\mathfrak{l})\right]_{P_{n}}$ is a factor.

$\delta$ ) Let $\omega=\int_{\Gamma} d \mu(\gamma) \omega_{\gamma}$ be the decomposition of $\omega$ obtained by diagonalizing $\mathfrak{B}$, then

$$
\left.\omega\right|_{\mathfrak{u}_{n}}=\left.\int_{\Gamma} d \mu(\gamma) \omega_{\gamma}\right|_{\mathfrak{u}_{n}} .
$$

This equation can for $A \in \pi_{\omega}\left(\mathfrak{U}_{n}\right)$ be read as

$$
\left(\Omega,[A]_{P_{n}} \Omega\right)=\int_{\Gamma} d \mu(\gamma) \omega_{\gamma}^{n}\left([A]_{P_{n}}\right),
$$

where $\omega_{\gamma}^{n}$ is the transpose of $\omega_{\gamma}$ under the isomorphism of $\pi_{\omega}\left(\mathfrak{U}_{n}\right)$ onto $\left[\pi_{\omega}\left(\mathfrak{U}_{n}\right)\right]_{P_{n}}$ (i.e. if $A \in \pi_{\omega}\left(\mathfrak{U}_{n}\right) \rightarrow[A]_{P_{n}}$ then $\left.\omega_{\gamma}^{n}={ }^{t} \phi_{n}\left(\omega_{\gamma}\right)\right)$. Equation (\#) therefore is the decomposition of a normal state on $\left[\pi_{\omega}\left(\mathfrak{U}_{n}\right)\right]_{P_{n}}$ into states on $\left[\pi_{\omega}\left(\mathfrak{U}_{n}\right)\right]_{P_{n}}$.

Since normality of $\omega_{\gamma}^{n}$ on $\left[\pi_{\omega}\left(\mathfrak{U}_{n}\right)\right]_{P_{n}}$ implies and is implied by normality of $\omega_{\gamma} \mid \mathfrak{x}_{n}$ we have by applying Theorem 5.1 the following

Corollary 5.2. For a $C^{*}$-algebra $\mathfrak{U}$ with a sequential separable factor funnel, a locally normal state $\omega$ thereupon and a commutative von Neumann algebra $\mathfrak{B}$ in $\pi_{\omega}(\mathfrak{U})^{\prime}$ the following three statements are equivalent:

i) $\omega=\int_{\Gamma} d \mu(\gamma) \omega_{\gamma}$ with $\omega_{\gamma}$ locally normal $\mu$ almost everywhere, where the decomposition is obtained by diagonalizing $\mathfrak{B}$. 
ii) The natural *-isomorphism

$$
\sum_{i=1}^{n} x_{i} b_{i} \mapsto \sum_{i=1}^{n} x_{i} \otimes b_{i}, \quad x_{i} \in\left[\pi_{\omega}\left(\mathfrak{U}_{n}\right)\right]_{P_{n}}, \quad b_{i} \in[\mathfrak{B}]_{P_{n}}
$$

extends to $a^{*}$-isomorphism of

$$
\left(\left[\pi_{\omega}\left(\mathfrak{U}_{n}\right)\right]_{P_{n}} \cup[\mathfrak{B}]_{P_{n}}\right)^{\prime \prime} \quad \text { onto } \quad\left[\pi_{\omega}\left(\mathfrak{U}_{n}\right)\right]_{P_{n}} \bar{\otimes}[\mathfrak{B}]_{P_{n}} .
$$

iii) The natural *-isomorphism

$$
\sum_{i} x_{i} b_{i} \mapsto \sum_{i} x_{i} \otimes b_{i}, \quad x_{i} \in\left[\pi_{\omega}\left(\mathfrak{U}_{n}\right)\right]_{P_{n}}, \quad b_{i} \in[\mathfrak{B}]_{P_{n}}
$$

entends to $a^{*}$-isomorphism of

$$
\left(\left[\pi_{\omega}\left(\mathfrak{U}_{n}\right)\right]_{P_{n}} \cup[\mathfrak{B}]_{P_{n}}\right)^{\prime \prime} \quad \text { onto } \quad \pi_{\omega}\left(\mathfrak{U}_{n}\right) \bar{\otimes} \mathfrak{B} .
$$

Because condition ii) in Theorem 5.1 is always fulfilled if $\mathfrak{M}$ is a type I factor on $\mathfrak{H}$ we have also

Corollary 5.3. For a $C^{*}$-algebra $\mathfrak{U}$ with a sequential separable type I factor funnel a decomposition of a locally normal state $\omega$ is always into locally normal states for any commutative Von Neumann algebra $\mathfrak{B}$ in $\pi_{\omega}(\mathfrak{U})^{\prime}$.

This corollary is also a consequence of [21] Proposition 4.3, because any type $I$ factor $\mathfrak{U}_{n}$ in the funnel contains the separable norm closed ideal I of the compact operators, that is $\sigma$-weakly-dense in $\mathfrak{U}_{n}$ and a locally normal state has the property that $\left\|\left.\omega\right|_{I}\right\|=\left\|\left.\omega\right|_{\mathfrak{u}_{n}}\right\|=1,[6]$.

One might attempt to extend these results to general locally normal states on a sequential separable factor funnel. This, however, is a rather difficult problem if we do not have some additional structure like in Theorem 5.1 and its Corollaries 5.2 and 5.3. As an illustration we want to conclude with an example of a locally normal state on a sequential separable factor funnel, whose central decomposition is not into locally normal states.

Let $G$ be a countably infinite discrete group such that every conjugacy class $C_{g}=\left\{C g C^{-1}: C \in G\right\}, g \in G$ is infinite except for the trivial one $C_{e}$. Such a group will be called an I.C.C.-group ([8], Chapitre III, $\S 7.7,[30]$, Chapter 4.2). Let $\mathfrak{M}(G)$ denote the von Neumann algebra generated by the left regular representation of $G . \mathfrak{M}(G)$ is known to be a $\mathrm{II}_{1}$ factor. Suppose $G$ has a decreasing sequence $\left\{G_{n}\right\}$ of subgroups such that:

(a) Each $G_{n}$ is an I.C.C.-group;

(b) $\bigcap_{n=1}^{\infty} G_{n}=A$ is an abelian subgroup such that $\mathfrak{M}(A)$ is a maximal abelian subalgebra of each $\mathfrak{M}\left(G_{n}\right)$.

If we take a free group with two generators $\alpha$ and $\beta$ as $G$ and a subgroup of $G$ generated by $\alpha$ and $\beta^{2^{n}}$ as $G_{n}$, then $G$ and $G_{n}$ satisfy the above conditions. 
Let $\mathfrak{S}$ be the Hilbert space $l^{2}(G)$ of all square summable functions on $G$. Each $\mathfrak{M}\left(G_{n}\right)$ acts on $\mathfrak{H}$. For each $n$, putting $\mathfrak{M}_{n}=\mathfrak{M}\left(G_{n}\right)^{\prime}$, we get an increasing sequence $\left\{\mathfrak{M}_{n}\right\}$ of separable factors. Let $\mathfrak{U}$ be the $C^{*}$-algebra generated by $\bigcup_{n=1}^{\infty} \mathfrak{M}_{n}$. Let $\Omega_{0}$ be the function in $\mathfrak{H}$ defined by

$$
\Omega_{0}(g)= \begin{cases}0 & g=e \\ 1 & \text { otherwise } .\end{cases}
$$

Then $\Omega_{0}$ is a cyclic vector for $\mathfrak{U}$. Therefore, the identity representation of $\mathfrak{U}$ is considered as the cyclic representation $\pi_{\omega}$ of $\mathfrak{U}$ induced by the state defined by

$$
\omega_{0}(x)=\left(x \Omega_{0} \mid \Omega_{0}\right), x \in \mathfrak{H} .
$$

Since we have

$$
\begin{aligned}
\mathfrak{U}^{\prime} & =\left(\bigcup_{n=1}^{\infty} \mathfrak{M}_{n}\right)^{\prime}=\bigcap_{n=1}^{\infty} \mathfrak{M}_{n}^{\prime} \\
& =\bigcap_{n=1}^{\infty} \mathfrak{M}\left(G_{n}\right)=\mathfrak{M}(A),
\end{aligned}
$$

$\pi_{\omega}$ is a type $I$ representation of $\mathfrak{U}$. The centre of $\pi_{\omega}(\mathfrak{U})^{\prime \prime}=\mathfrak{U} \mathfrak{U}^{\prime \prime}$ coincides with $\mathfrak{M}(A)$. If we decompose $\omega$ with respect to the centre $\mathfrak{M}(A)$ of $\pi_{\omega}(\mathfrak{l})^{\prime \prime}$, then the restriction $\left.\omega\right|_{\mathfrak{M}_{n}}$ of $\omega$ to $\mathfrak{M}_{n}$ is also decomposed with respect to $\mathfrak{M}(A)$. But $\mathfrak{M}(A)$ is maximal abelian in $\mathfrak{M}_{n}^{\prime}=\mathfrak{M}\left(G_{n}\right)$, so that the restriction of the components to $\mathfrak{M}_{n}$ cannot be normal because $\mathfrak{M}_{n}$ is not of type $I$. Indeed $\mathfrak{M}_{n}$ is a type $I I$ factor with $\left(\mathfrak{M}_{n}\right)_{*}$ separable; hence a normal state on $\mathfrak{M}_{n}$ gives rise to a cyclic representation on a separable Hilbert-space (cf. $\S 3$, Corollary 1) and this representation is normal and faithful $[10,17,35]$, and is therefore type $I I$. This implies that the central decomposition, in this case, cannot be accomplished in the frame of locally normal states. To argue this last point, suppose that one would have

$$
\left.\omega\right|_{\mathfrak{M}_{n}}=\left.\int_{\Gamma} d \mu(\gamma) \omega_{\gamma}\right|_{\mathfrak{M}_{n}}=\int_{\omega_{n}(\Gamma)} d v\left(\phi^{n}\right) \phi^{n},
$$

where $\phi^{n}$ is $v$-a.e. normal on $\mathfrak{M}_{n}$ (i.e. $v\left(\omega_{n}(\Gamma) \backslash N\right)=1$ and $\phi^{n} \in \omega_{n}(\Gamma) \backslash N$ is normal on $\mathfrak{M}_{n}$ with $N$ the appropriate null-set).

Since $\mathfrak{M}(A)$ is maximal abelian in $\mathfrak{M}_{n}^{\prime}=\mathfrak{M}\left(G_{n}\right)$ we know (cf. [30]) that $\left(v_{0}\right)_{*}\left(\omega_{n}(\Gamma) \backslash N\right)=0$ (where $\left(v_{0}\right)_{*}$ is the inner Baire measure obtained from the Baire contraction of the regular Borel measure $v$ ) because $\omega_{n}(\Gamma) \backslash N$ belongs to the hereditary $\sigma$-ring ${ }^{5}$ generated by the Baire sets. $\left(\mathfrak{M}_{n}\right)_{*}$ is separable as a Banach space hence $S\left(\mathfrak{M}_{n}\right)$ is separable in the $\sigma\left(\mathfrak{M}_{n}^{*}, \mathfrak{M}_{n}\right)$ topology and therefore

$$
v\left(\omega_{n}(\Gamma) \backslash N\right)=v_{*}\left(\omega_{n}(\Gamma) \backslash N\right)=0,
$$

where $v_{*}$ is the inner Borel measure. This means that we obtained a contradiction because we assumed $v\left(\omega_{n}(\Gamma) \backslash N\right)=1$.

\footnotetext{
${ }^{5}$ cf. P. R. Halmos, Measure Theory for terminology.
} 
Acknowledgements. The authors are very grateful that their cooperation was made possible by an invitation of the first named author to the C.N.R.S. in Marseille by D. Kastler and an invitation to the Institute of Theoretical Physics in Groningen by N. M. Hugenholtz. The second named author thanks the Netherlands Organisation for Pure Research (Z.W.O.) for financial support during part of this work.

\section{References}

1. Bourbaki, N.: Intégration. Paris: Hermann et Cie 1952.

2. Choquet, G.: Lectures on analysis. Edited by J. Marsden, T. Lance and S. Gelbart. New York, Amsterdam: W. A. Benjamin inc. 1969.

3. Dell'Antonio, G.F., Doplicher, S., Ruelle, D.: A theorem on canonical commutation and anti-commutation relations. Commun. math. Phys. 2, 223-230 (1966).

4. Dixmier, J.: Sur certains espaces considérés par M. H. Stone. Summa Brasil. Math. t2., fasc. 11, 151-182 (1951).

5. Dixmier, J.: Algèbres quasi-unitaires. Comm. Math. Helv. 26, 275-322 (1952).

6. Dixmier, J.: Les fonctionelles linéaires sur l'ensemble des opérateurs bornés d'un espace de Hilbert. Ann. Math. 51, 387-408 (1950).

7. Dixmier, J.: Les $C^{*}$-algèbres et leurs representations. Paris: Gauthier-Villars 1964.

8. Dixmier,J.: Les algèbres d'opérateurs dans l'espace Hilbertien. Paris: GauthierVillars 1969.

9. Effros, E. G.: Structure in simplexes. Acta Mathematica 117, 103-121 (1967).

10. Feldman, J., Fell, J. M. G.: Separable representations of rings of operators. Ann. Math. 65, 241-249 (1957).

11. Haag, R., Hugenholtz, N.M., Winnink, M.: On the equilibrium states in quantum statistical mechanics. Commun. math. Phys. 5, 215 (1967).

12. Haag, R., Kadison, R.V., Kastler, D.: Nets of $C^{*}$-algebras and classification of states. Commun. math. Phys. 16, 81 (1970).

13. Hille,E., Phillips, R.S.: Functional analysis and semi-groups. Amer. Math. Soc. Colloq. Publ. Vol. XXXI (1957).

14. Hugenholtz, N.M., Wieringa, J.D.: On locally normal states in quantum statistical mechanics. Commun. math. Phys. 11, 183-197 (1969).

15. Jadczyk, A.Z.: On some groups of automorphisms of von Neumann algebras with a cyclic and separating vector. Commun. math. Phys. 13, 142-154 (1969).

16. Kadison, R.V., Ringrose, J. R.: Derivations and automorphisms of operator algebras. Commun. math. Phys. 4, 32 (1967).

17. Kaplansky, I.: Representations of separable algebras. Duke Math. J. 19, 219 (1952).

18. Kallmann, R.R.: Unitary groups and automorphisms of operator algebras. Amer. J. Math. 91, 785-806 (1969).

19. Kastler,D., Robinson,D.W.: Invariant states in statistical mechanics. Commun. math. Phys. 3, 151-180 (1966).

20. Lanford, O., III: Quantum spin systems. In: Cargèse Lectures in Physics, Vol. 4, ed. D. Kastler, New York: Gordon and Breach 1970.

21. Lanford, O. III., Ruelle, D.: Integral representations of invariant states on $B^{*}$-algebras. Journ. Math. Phys. 8, 1460-1463 (1967).

22. Manuceau, J.: $C^{*}$-algèbre de relations de commutation. Ann. de l'instit. H. Poincaré 2 , 139 (1968).

23. Naimark, M. A.: Normed rings. Groningen: Noordhoff 1964.

24. Phelps, R. R.: Lectures on Choquet's theorem. Princeton, N.J.: Van Nostrand 1966.

25. Pukansky, L.: On the theory of quasi-unitary algebras. Acta Sci. Math. Szeged 16, $103-121$ (1955). 
26. Robinson, D. W.: Statistical mechanics of quantum spin systems II. Commun. math. Phys. 7, 337 (1968).

27. Rocca,F., Testard, D., Sirugue, M.: On a class of equilibrium states under the KuboMartin-Schwinger condition I Fermions. Commun. math. Phys. 13, 319 (1969).

28. Ruelle, D.: States of physical systems. Commun. math. Phys. 3, 133-150 (1966).

29. Ruelle,D.: Symmetry Breakdown in statistical mechanics. In: Cargèse Lectures in Physics Vol. 4, Ed. D. Kastler. New York: Gordon and Breach 1970.

30. Sakai, S.: $C^{*}$-algebras and $W^{*}$-algebras. Berlin-Heidelberg-New York: Springer 1971.

31. Sherman, S.: Order in operator algebras. Amer. J. Math. 73, 227-232 (1951).

32. Sirugue, M., Winnink, M.: Constraints imposed upon a state of a system that satisfies the K.M.S. boundary condition. Commun. math. Phys. 19, 161-168 (1970).

33. St $\varnothing$ rmer, E.: Large groups of automorphisms of $C^{*}$-algebras. Commun. math. Phys. $\mathbf{5}$, $1-22(1967)$.

34. Takesaki, M.: On the conjugate space of operator algebra. Tohôku Math. J. 10, 194-203 (1958).

35. Takesaki, M.: On the non-separability of singular representations of operator algebras Kodai. Math. Sem. Repts. 12, 102 (1960).

36. Takesaki, M.: On the cross-norm of the direct product of $C^{*}$-algebras. Tohôku Math. J. 16, 111-122 (1964).

37. Takesaki, M.: Tomita's theory of modular Hilbert algebras and its applications. Lectures in Math. 128. Berlin-Heidelberg-New York: Springer 1970.

38. Testard,D.: Les états quasi-libres du champ de Bosons considerés, comme états d'equilibre à l'aide de la condition de Kubo, Martin et Schwinger, Thesis, Marseille 1970.

39. Tomita, M.: Quasi-standard von Neumann algebras. Kyushu University preprint.

40. Winnink, M.: An application of $C^{*}$-algebras to quantum statistical mechanics of systems in equilibrium. Thesis Groningen 1968.

41. Winnink, M.: Algebraic consequences of the K.M.S. boundary condition. In: Cargèse Lectures in Physics, Vol. 4. Ed. D. Kastler. New York: Gordon and Breach 1970.

Masamichi Takesaki

Department of Mathematics

University of California

Los Angeles, Cal. 90024, USA
Marinus Winnink

Institute for Theoretical Physics

University of Groningen

Groningen, The Netherlands 